\title{
Sparse Weakly Supervised Models for Object Localization in Road Environment
}

\author{
Valentina Zadrija ${ }^{\mathrm{a}}$, Josip Krapac ${ }^{\mathrm{b}, *}$, Siniša Šegvića Jakob Verbeek $^{\mathrm{c}}$ \\ ${ }^{a}$ University of Zagreb, Faculty of Electrical Engineering and Computing, Croatia \\ ${ }^{b}$ Mobius Labs, Berlin, Germany \\ ${ }^{c}$ Univ. Grenoble Alpes, Inria, CNRS, Grenoble INP, LJK, 38000 Grenoble, France
}

\begin{abstract}
We propose a novel weakly supervised localization method based on Fisher-embedding of low-level features (CNN, SIFT), and model sparsity at the component level. Fisher-embedding provides an interesting alternative to raw lowlevel features, since it allows fast and accurate scoring of image subwindows with a model trained on entire images. Model sparsity reduces overfitting and enables fast evaluation. We also propose two new techniques for improving performance when our method is combined with nonlinear normalizations of the aggregated Fisher representation of the image. These techniques are i) intra-component metric normalization and ii) first-order approximation to the score of a normalized image representation. We evaluate our weakly supervised localization method on real traffic scenes acquired from driver's perspective. The method dramatically improves the localization AP over the dense nonnormalized Fisher vector baseline (16 percentage points for zebra crossings, 21 percentage points for traffic signs) and leads to a huge gain in execution speed ( $91 \times$ for zebra crossings, $74 \times$ for traffic signs).
\end{abstract}

Keywords: Object localization, Weak supervision, Fisher Vectors, Sparse models, Convolutional features, Geographic information system (GIS), OpenStreetMap

\section{Introduction}

Detecting the presence of objects in images and recovering their locations are very important yet still open computer vision problems. These problems are often jointly addressed by applying a localization model at many image locations, and reporting objects where a positive response was obtained. Most successful representatives of this approach employ strong supervision at the training stage, which requires that each training image be annotated with accurate object locations. However, annotating object locations is expensive due to significant human labeling effort involved, even if a simple location model is used (e.g. bounding box). This is especially the case in realistic scenarios where thousands of annotations are required to achieve top performance. Annotation is particularly difficult when the objects of interest are small, since near to pixel-level annotation accuracy may be required for best results.

In order to alleviate the effort of full annotation, many recent approaches attempt to solve the localization problem in a weakly-supervised manner (cf. e.g. [1, 2, 3]). In this setting, training images are annotated only with class labels. The training procedure is supposed to train the localization model without knowing the object locations. At the test time, however, bounding boxes have to be predicted for each learned object class as in the strongly supervised case. This can be useful even if the recovered object classifier is not particularly fast, since the recovered object locations can be used to train a more efficient localization model in a strongly supervised fashion.

Weakly supervised training of object classifiers is a daunting task in most realistic scenarios. Even if we assume only one object in each positive image (which is not the case in our experiments), an exhaustive search would have to consider $\mathrm{W}^{\mathrm{T}}$ hypotheses, where $\mathrm{W}$ is the number of image windows and $\mathrm{T}$ is the number of training images. This complexity may be decreased by sampling [4], clustering [5] or employing bottom-up location proposals based on

\footnotetext{
${ }^{*}$ Corresponding author

Email address: josip.krapac@gmail .com (Josip Krapac)
} 
trained segmentation [6, 3] or objectness cues [7, 8, 9, 10, 11]. However all these approaches risk to miss some objects at the selection stage, which may invalidate all subsequent efforts.

A more conservative approach relies on classifiers able to detect the object presence in a larger image context. Such classifiers can be trained on positive images [12] or image regions [6] and then subsequently applied to recover or gradually improve the object localization. Some of the previous work along these lines has been based on BoW histograms [6, 12] and Fisher vectors coupled with bottom-up location proposals [3]. Recently, several researches have proposed approaches based on convolutional classifiers and end-to-end training [13, 14, 2].

In this paper we present a novel weakly-supervised object localization method based on Fisher vectors and model sparsity at the component level. Earlier accounts of this research appeared in [15, 16]. We extend that work in several ways:

- we present an end-to-end case study of road-environment mapping by training and evaluating our localization pipeline on images collected from crowd-sourced GPS labels (Sections 4.5 .2 ),

- we propose improved methods for generating location responses from top rated patches (Section 3.6),

- we improve support for our claims by presenting experiments on convolutional features (Sections 5.2, 5.4),

- we discuss comparative advantages of the sparse models against the dense ones for localization and classification tasks (Section 5.5).

In section 2 we argue that our method has important advantages with respect to other weakly-supervised localization approaches. These advantages arise since: i) Fisher vectors pool better than raw features due to ability to preserve and enhance unusual detail, ii) component-level sparsity enables fast evaluation and reduces overfitting, iii) we enable non-linear normalizations by intra-component normalization and approximated patch scoring and iv) the method does not require bottom-up location proposals. Section 3 presents details of the proposed method: patch-level Fisher vector embedding (Section 3.1), sparse localization models (Section 3.3) and first-order approximation of the patch contribution (Section 3.4), efficient determination of the patch-level response (Section 3.5) and the recovery of bounding boxes (Section 3.6. Section 4 presents an effective semi-automatic procedure for collecting training images by exploiting crowd-sourced GPS labels. Section 5 presents an experimental validation of the proposed method on two datasets pertinent to the problem of automated road environment mapping. The datasets contain very small objects with much intra-class variation, in front of information-abundant background. We achieve good localization performance, comparable to strongly supervised approaches, while using a sparse model that accesses only a small fraction of the visual representation.

\section{Related work}

Most of existing weakly supervised localization approaches mitigate the computational complexity of weak supervision by relying on bottom-up location proposals. These approaches typically adopt the following iterative structure: i) train a discriminative model on the current guess of object locations in positive images, ii) use that model to select a better guess for the next iteration. This scheme optimizes a criterion that at least one (or exactly one [1, 7, , , 12]) object is found in each positive image and that no objects are found in negative images. The optimization has been formulated as multiple-instance learning [6, 7, 4, 3, 1, 9] or end-to-end learning [10, 11]. All of these approaches may completely overlook small objects in training images, especially in traffic scenes with rich backgrounds. In our preliminary experiments, a popular objectness algorithm [1] consistently failed to produce accurate traffic sign locations in top 2000 proposals. Additionally, this kind of optimization is computationally very intensive, which complicates training on large datasets.

Another possible approach is to start optimization by discriminating entire (or almost entire) images and then gradually zoom onto object locations through iteration. One way to formulate this iteration is to present object locations as latent variables in a deformable part model framework [1]. Another approach would be to construct an integral image of the patch scores and then find regions which maximize the classification score [12]. Both of these approaches do not require bottom-up location proposals, however they are prone to convergence issues, while not being able to handle training images with multiple objects. Classification of entire images has also been used 
to kick-off subsequent MIL optimization [3]. In each iteration, false positives of the current model are chosen as future negative samples, while future positive samples are set to top-scored bottom-up location proposals. However, as above, reliance on bottom-up location proposals may represent a liability in datasets with small objects.

Several recent approaches [13, 14, 2, 17] train weakly supervised localization models by exploiting deep convolutional architectures without bottom-up proposals and MIL-like iterations. These architectures are pre-trained on ImageNet and adapt the convolutional architecture for localization by converting fully connected layers to convolutional ones. The approach [14] completes the pipeline with global average pooling and fine-tunes all parameters with classification loss. The approach [13] avoids fine tuning by appending two convolutional adaptation layers and training them for classification of max-pooled scores on the target dataset. These approaches are attractive since they determine patch scores in a single forward pass while retaining the capability for end-to-end learning. However, they require joint training for all classes while our approach requires only the training of a distinct linear classifier for each class. Additionally, pooled convolutional features offer sub-optimal performance in popular architectures with a fully-connected back-end [18]. Hence [2] propose to perform localization by iteratively applying a fully-connected layer to down-sampled convolutional representations extracted within the active location hypotheses. The iteration is formulated as a beam-search under premise that better centered objects give rise to higher classification scores. Unfortunately, this is computationally much more expensive than the approaches based on pooling [13, 14], since it requires many forward passes through the network (additionally, beam search is not guaranteed to converge). Recently, Zhu et al [17] propose to generate objectness maps by iteratively accumulating confidence at the nodes that have high dissimilarity with their surroundings. The obtained objectness maps are then projected back, and further jointly optimized with network parameters.

Similarly to [13, 14], we also avoid bottom-up proposals by pooling local features. Our approach is perfectly suitable for end-to-end learning, although, in this work, we show experimental results exclusively on raw convolutional features provided by the public parameterization of the architecture VGG-19 [18]. However, different from [13, 14, 17], we avoid losing classification power with respect to the fully-connected case, by embedding low-level features (CNN, SIFT) into the Fisher space [19, 3, 20]. Aggregation of Fisher-embedded convolutional features has a very similar (if not larger) representative power as classical convolutional networks terminated with fully-connected layers [21]. Thus we obtain fair performance comparable to [2] and fast execution speed comparable to [13, 14, 17] in spite of the high dimensionality of Fisher representation. We succeed to keep computational complexity tractable by reinforcing a group-sparse classification model [22, 16, 23] and exploiting the first-order approximation of the patch contribution to the normalized Fisher vector of the image [16].

Recent work on zebra crossing localization [24, 25] is based on ad-hoc hand-crafted features of appearance and shape. Line segments have been used in [24] to detect zebra crossings in corresponding Google satellite and streetview images acquired over a $1.6 \mathrm{~km}^{2}$ area in San Francisco ${ }^{1}$ A dataset of aerial images spanning across several countries has been proposed in [25], along with a detection approach based on HOG and LBPH features. Both approaches specialize for zebra crossings and require aerial imagery. On the other hand, our method is applicable to perspective views of various object classes (as shown in experiments on traffic signs and zebra crossings), while requiring only image-wide labels and no feature engineering whatsoever.

\section{The proposed weakly supervised localization method}

We present a method suitable for learning a localization model from crowd-sourced image-wide labels and georeferenced video. The method is based on Fisher-embedding of low-level features [28] and group-sparse [29] classification models (see Figure 11). We train a sparse model on Fisher vectors of entire images and use non-linear normalizations [30] to improve the classification performance. The obtained classifier is applied at all image locations to identify patches which contribute to the classification decision. A novel first-order approximation of the patch-contribution to the classification score ensures inter-operability with normalizations implied by improved Fisher vector [30]. We succeed to achieve near real-time performance due to fast evaluation of a group-sparse localization model. Localization responses (bounding boxes or convex hulls) are finally inferred by clustering positive patches, as illustrated in Figure 1 .

${ }^{1}$ In the process of acquiring the weakly supervised dataset for our experiments, we have contacted Google regarding the San Francisco dataset [26]. The response was negative so we acquired our dataset from other sources [27]. 


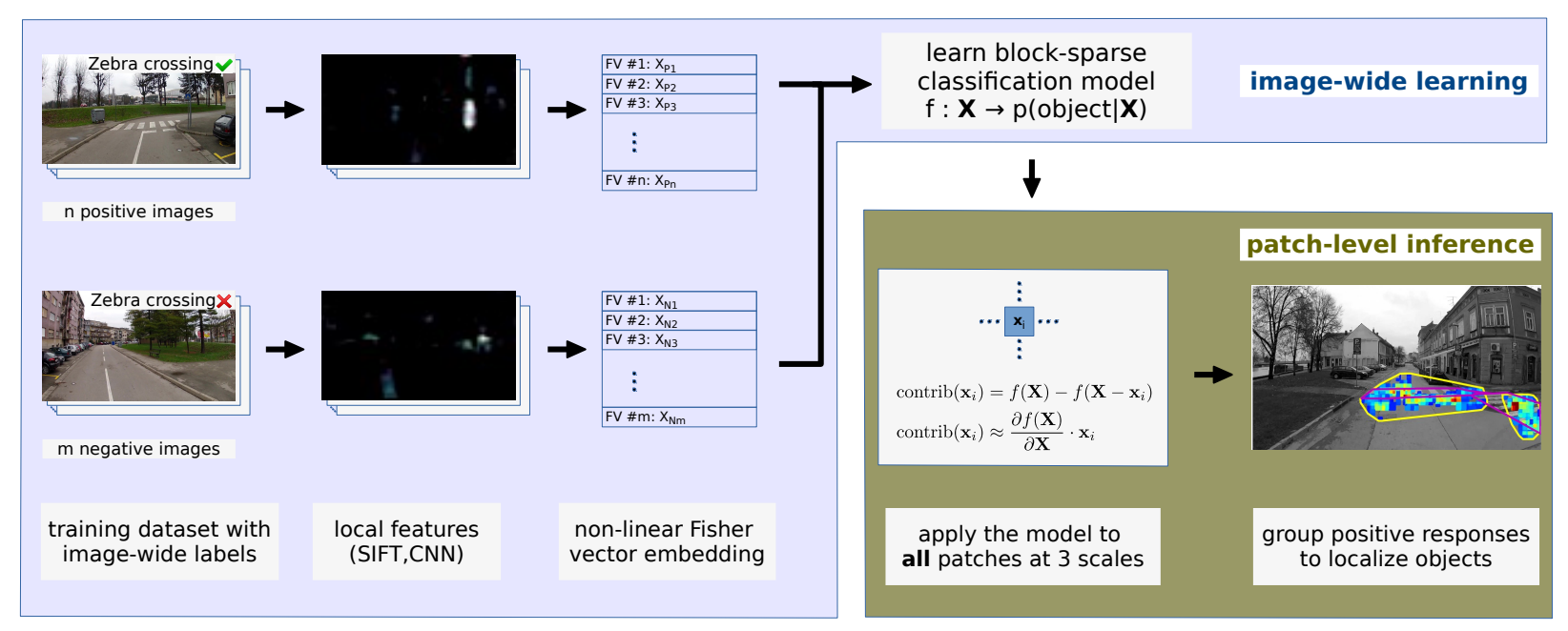

Figure 1: Overview of the proposed weakly supervised pipeline for road-related object mapping. Please refer to the text for a more complete description.

\subsection{Fisher vectors for weakly supervised localization}

We regard images as orderless bags of patch descriptors (e.g. SIFT, convolutional features) to which we fit a generative Gaussian mixture model (GMM) $\theta=\left\{\alpha_{i}, \boldsymbol{\mu}_{i}, \boldsymbol{\sigma}_{i}\right\}_{i=1}^{K}$. Such model can be viewed as a visual vocabulary while its components can be referred to as visual words. The probability density function of a patch descriptor $\mathbf{x}$ can be stated as $p(\mathbf{x} \mid \theta)$. At this point, we may represent $\mathbf{x}$ as the gradient of the log-likelihood with respect to model parameters $\theta$ :

$$
\mathrm{U}(\mathbf{x} \mid \theta)=\nabla_{\theta} \log p(\mathbf{x} \mid \theta) .
$$

The score $\mathrm{U}(\mathbf{x} \mid \theta)$ succinctly describes the relation of the data point with respect to the parameters of the generative model [31]. We obtain the Fisher vector $\Phi(\mathbf{x} \mid \theta)$ by decorrelating the score [32]:

$$
\Phi(\mathbf{x} \mid \theta)=\mathbf{F}(\theta)^{-0.5} \cdot \mathrm{U}(\mathbf{x} \mid \theta) \text {, where } \mathbf{F}(\theta)=\mathrm{E}_{\mathbf{x}}\left[\mathrm{U}(\mathbf{x} \mid \theta) \mathrm{U}^{\top}(\mathbf{x} \mid \theta)\right] .
$$

The resulting representation captures first and second order statistics with respect to GMM components, and as such corresponds to a quadratic function operating on low-level descriptors. Therefore, a linear classifier in the embedded space corresponds to a smooth piecewise quadratic decision boundary in the original space. However, besides being a quadratic kernel, the FV representation exhibits the following additional properties which make it especially suitable for weakly supervised localization:

1. additivity for $\mathbf{x}_{i}$ i.i.d.: $\Phi\left(\left\{\mathbf{x}_{i}\right\} \mid \theta\right)=\sum_{i} \Phi\left(\mathbf{x}_{i} \mid \theta\right)$

2. vanishing expectation: $\mathrm{E}_{\mathbf{x}}[\Phi(\mathbf{x} \mid \theta)]=0$,

3. unit covariance: $\mathrm{E}_{\mathbf{x}}\left[\Phi(\mathbf{x} \mid \theta) \Phi^{\top}(\mathbf{x} \mid \theta)\right]=\mathbf{I}$.

We see that the representation of the whole image corresponds to the sum of patch representations. This allows to reverse the stages of pooling and scoring and to apply an image-wide linear classification model to locate patches responsible for the image label. Additionally, the FV representation attenuates the background information due to vanishing expectation. Equation (1) suggests that unusual data points "surprise" the generative model and therefore exert a strong influence to the aggregated representation. Thus, small distinctive objects stand a better chance to be noticeable in the image representation than in other aggregation approaches. 


\subsection{Non-linear normalizations of Fisher vectors}

In this paper we use improved Fisher vectors [30] which involve power and metric normalizations of the image representation. The power normalization (signed square-rooting) is applied to each dimension $X_{d}$ of the Fisher vector as $s\left(X_{d}\right)=\operatorname{sign}\left(X_{d}\right)\left|X_{d}\right|^{\rho}$, with $0<\rho<1$. The power normalization can be understood in terms of a positive semi-definite kernel function $K\left(\mathbf{X}, \mathbf{X}^{\prime}\right)=\left\langle\mathbf{s}(\mathbf{X}), \mathbf{s}\left(\mathbf{X}^{\prime}\right)\right\rangle$, where computing the power norm "un-sparsifies" the vector $\mathbf{X}$ and makes it more suitable for comparison with the dot product. The power normalization also accounts for the assumption that the low-level descriptors are i.i.d. [28]. The metric normalization projects the Fisher vector onto the unit hyper-sphere by dividing it with $\sqrt{n(\mathbf{X})}$ where $n(\mathbf{X})=\sum_{d} s\left(X_{d}\right)^{2}$. This accounts for the fact that different images contain different amounts of background information [28].

In our work, we also experiment with the intra-component normalization [33, 23], where the $\ell_{2}$ normalization is separately applied to the parts of the Fisher vector corresponding to different GMM components. In order to formally define the intra-normalized Fisher vector of the image, we use $\mathbf{X}^{k}$ to denote the part corresponding to the $k$-th visual word and write the corresponding $\ell_{2}$ norm as $n\left(\mathbf{X}^{k}\right)$. Hence, given the GMM vocabulary with $K$ components, the intra-component normalized FV corresponds to:

$$
\frac{1}{\sqrt{K}} \cdot\left[\frac{\mathbf{s}\left(\mathbf{X}^{1}\right)}{n\left(\mathbf{X}^{1}\right)} \frac{\mathbf{s}\left(\mathbf{X}^{2}\right)}{n\left(\mathbf{X}^{2}\right)} \ldots \frac{\mathbf{s}\left(\mathbf{X}^{k}\right)}{n\left(\mathbf{X}^{k}\right)}\right] .
$$

The normalization with $\sqrt{K}$ guaranties that $\|\mathbf{X}\|=1$, when at least one patch is assigned to each GMM component.

\subsection{Sparse models for classification and localization}

Given the set of Fisher vectors $\mathbf{X}_{\mathbf{i}}$ and the corresponding image-wide labels $y_{i} \in\{-1,1\}$, we train a linear classifier $\mathbf{w}$ by minimizing the following regularized logistic loss function:

$$
\ell(\mathbf{w}, \mathbf{X}, \mathbf{y})=\sum_{i=1}^{N} \log \left(1+\exp \left(-y_{i} \cdot \mathbf{w}^{\top} \mathbf{X}_{i}\right)\right)+\lambda \cdot \mathcal{R}(\mathbf{w}) .
$$

In the above equation, $N$ denotes the number of images in the training subset, $\mathcal{R}(\mathbf{w})$ denotes the regularization function, while $\lambda$ represents the trade-off between the loss and the regularization. The most widely adopted choices for $\mathcal{R}(\mathbf{w})$ include $\ell_{2}(\mathbf{w})=\sum_{j} w_{j}^{2}$, and $\ell_{1}(\mathbf{w})=\sum_{j}\left|w_{j}\right|$. The $\ell_{1}$ regularization is of particular interest since it favours sparse models [34, 35] in which the majority of coefficients is zero. Such bias expresses a prior that the majority of image representation does not directly correspond to an instance of the considered object class. This kind of prior is very desirable in weakly supervised localization of small objects, since it discourages overfitting to soft contextual cues and allows efficient patch scoring.

However, $\ell_{1}$ regularization ignores two important pieces of prior information specific to the classification of Fisher vectors. First, the contributions of particular object classes is typically concentrated in parts of the FV representation corresponding to only a few words from the visual dictionary. This happens because feature extraction algorithms are designed or trained in a way that low-level features found at particular object classes group in the feature space. Second, the quickest way to process an image patch in our framework is to reject it immediately after the soft-assign stage, by noticing that the model coefficents are zeros at all components to which the patch contributes. Thus we see that instead of opting for unstructured flat sparsity induced by $\ell_{1}$ regularization, it makes much more sense to prefer structured component-level sparsity where only coefficients corresponding to a few selected components are different than zero [22, 16, 23]. This kind of sparsity may conveniently be encouraged by supplying a regularization function defined as a sum of $\ell_{2}$ regularization within components and $\ell_{1}$ regularization across components:

$$
\ell_{2,1}(\mathbf{w})=\sum_{k} \ell_{2}\left(\mathbf{w}^{k}\right)
$$

\subsection{From image classification to patch-level scores}

Let $f(\mathbf{X})$ denote the classification score of the image representation $\mathbf{X}$. Then, the contribution of patch $\mathbf{x}$ to the overall image score can be expressed as:

$$
p c_{\text {direct }}(\mathbf{x})=f(\mathbf{X})-f(\mathbf{X}-\mathbf{x}) .
$$


In the case of a linear classification model and un-normalized FV representation, we have $f_{\text {linear }}(\mathbf{X})=\mathbf{w}^{\top} \mathbf{X}$. Therefore, we can reverse the scoring and sum-pooling operations and express the patch contribution as a simple dot product:

$$
p c_{\text {linear }}(\mathbf{x})=f_{\text {linear }}(\mathbf{X})-f_{\text {linear }}(\mathbf{X}-\mathbf{x})=\mathbf{w}^{\top} \mathbf{x}
$$

In the case of improved FV [30], the linear image score can be expressed as:

$$
f(\mathbf{X})=\mathbf{w}^{\top} \mathbf{s}(\mathbf{X}) / \sqrt{n(\mathbf{X})}
$$

We see that non-linear normalizations described in Section 3.1 invalidate additivity of the Fisher representation and make the linear decomposition impossible: $\mathbf{w}^{\top} \mathbf{s}(\mathbf{X}) / \sqrt{n(\mathbf{X})} \neq \sum_{\mathbf{X}} \mathbf{w}^{\top} \mathbf{s}(\mathbf{x}) / \sqrt{n(\mathbf{x})}$. Instead the patch contribution may be computed directly, as in (6). This requires the following operations to be applied at each image patch: (i) subtracting the patch representation $\mathbf{x}$ from the image representation $\mathbf{X}$, (ii) applying the power and $\ell_{2}$ normalizations onto the result, (iii) scoring with the model $\mathbf{w}$ and (iv) subtracting the obtained score from the image score. Unfortunately, this procedure is computationally very complex which makes its application to all image patches impractical. We therefore propose a first-order approximation which corresponds to taking the dot-product between the un-normalized patch representation and the gradient of the normalized image score.

We now derive the gradient of the classification score of the normalized image representation w.r.t. non-normalized patch representation $\mathbf{x}$. The partial derivative of the score w.r.t. an element of the non-normalized patch $x_{d}$ is given by $\partial f(\mathbf{X}) / \partial x_{d}=\partial f(\mathbf{X}) / \partial \mathbf{X} \cdot \partial \mathbf{X} / \partial x_{d}$. The derivative of the non-normalized image representation w.r.t. the d-th element of the patch FV corresponds to the vector with all zero elements except the d-th which is equal to one. Hence, the gradient w.r.t. the patch element $x_{d}$ is equal to the gradient w.r.t. image element $X_{d}$ :

$$
\frac{\partial f(\mathbf{X})}{\partial x_{d}}=\frac{\partial f(\mathbf{X})}{\partial X_{d}}=\frac{\rho\left|X_{d}\right|^{\rho-1}}{\sqrt{n(\mathbf{X})}}\left(w_{d}-\frac{s\left(X_{d}\right) f(\mathbf{X})}{\sqrt{n(\mathbf{X})}}\right) .
$$

For more details regarding the gradient derivation, the reader can refer to Appendix $\mathrm{A}$ The above expression is undefined in cases where $X_{d}=0$ (a rare case since the full image FV are dense). In such cases, we set the derivative to zero to ignore the impact of such dimensions.

In the case of intra-component normalization, the classification score is a sum of per-component classification scores: $f(\mathbf{X})=\sum_{k} f_{k}\left(\mathbf{X}^{k}\right)$. Since the $f_{k}\left(\mathbf{X}^{k}\right)$ have precisely the same form as $f(\mathbf{X})$ above, we can compute the gradients in the same manner, per each component.

\subsection{Efficient patch scoring with a sparse model}

The complexity of patch scoring can be subdivided into the following three stages with similar computational complexity: i) computing the soft-assign $p(k \mid x)$, ii) computing the $\mathrm{FV}$, and iii) determining the patch contribution by (6), 77), or (9). In this paper, we consider efficient implementation of the latter two stages for CPU architectures (efficient soft-assign is addressed in [36]). In the naive implementation, both of these two stages are $O(N K D)$ where $\mathrm{N}$ is the number of patches, $\mathrm{K}$ is the number of components, while $\mathrm{D}$ is the raw feature dimensionality. We reduce that complexity by exploiting two kinds of sparsity. The soft-assign sparsity refers to the fact that the GMM posterior is very sparse: a majority of patches are dominantly assigned to only one GMM component. The model sparsity indicates that our models typically reference only a tiny part of the representation as described in Section 3.3 .

First, we find GMM components with significant soft-assign $p(k \mid x)>1 / K$ and denote their number with $K_{s}$. Due to soft-assign sparsity we typically have $K_{s} \ll K$. Second, we identify $K_{w}$ non-zero blocks of the model $w$, where blocks correspond to the GMM components. In the case of the group-sparse regularizer (5), $K_{w}$ is directly influenced by the amount of regularization and we typically have $K_{w} \ll K$. At this point we can reject the patches that are not assigned to any of the $K_{w}$ selected components. This reduces the number of patches from $N$ to $N^{\prime}$ and, depending on the abundance of the object class, may result in very large speedups $\left(N^{\prime} \ll N\right)$, similarly to the effects of the first few stages of a cascaded classifier [37]. For the remaining $N^{\prime}$ patches we need to compute only the parts of the Fisher vector which correspond to the intersection of $K_{s}$ assigned components and $K_{w}$ components incident to the model. Therefore, we need to compute Fisher vectors and patch contributions only for at $\operatorname{most} K^{\prime}=\min \left(K_{s}, K_{w}\right)$ components. Total complexity of these two stages for all image patches is $O\left(N^{\prime} K^{\prime} D\right)$, corresponding to a tiny fraction of the original complexity. 
This efficient procedure is equally applicable for linear scoring with the model $\mathbf{w}$ and the gradient (9). The proposed procedure can also be applied for direct scoring (6) of intra-normalized patch representations. In this case the sparsity of patch representation and the locality of intra-normalization ensure that only the patches generated by the $K_{w}$ selected components may result in non-zero scores.

Please note that the efficient procedure is not exact in the case of global normalization (8) and direct scoring (6), since the contribution outside the selected $K_{w}$ components alters the representation norm. Hence, this combination requires exhaustive evaluation of the patch score which results in extraordinary long execution times as we shall see in the experiments.

\subsection{From patches to objects}

We extract low-level patches on several scales, embed them into high-dimensional FV space and compute their contributions to the overall image score as described in previous sections. We explore two different approaches for generating localization responses from top-rated patches: (i) independent processing of each particular scale, and (ii) combining per-scale responses in a unified heat map.

\subsubsection{Per scale approach}

We build a spatial graph by connecting $T$ top-rated patches on each particular scale which overlap more than $P \%$. Localization responses are generated as a union of patches assigned to the particular connected component. Connected components with less than $N$ patches are removed from consideration. The main motivation behind this approach is to prevent co-occurring background patches of different sizes to be recognized as objects. This approach has been used to generate localization responses in experiments presented in Section 5.3, which contains referent values for parameters $T, P$ and $N$.

\subsubsection{Multi-scale heat map approach}

We compute per-scale patch contributions at the resolution of the low-level features, and up-sample them to the resolution of the original image by nearest neighbour interpolation. Let $f\left(x_{i} \mid c, s\right)$ denote the patch score at the pixel $x_{i}$ for the class $c$ obtained by up-sampling the scores of patches at the scale $s$. The cumulative score $f\left(x_{i} \mid c\right)$ at each pixel $x_{i}$ is then computed as:

$$
f\left(x_{i} \mid c\right)=\sum_{s} f\left(x_{i} \mid c, s\right) .
$$

In order to generate localization responses from the obtained unified heat map, we consider pixels with a score greater than some threshold $T$. In practice, we set $T$ to be an average of all pixels with a positive score, i.e. $T=1 / P \cdot \sum_{i} f\left(x_{i} \mid c\right)$, such that $f\left(x_{i} \mid c\right)>0$, where $P$ denotes the number of such pixels. We group the selected pixels into connected components and generate localization polygons as convex hulls of pixels in a particular component. This approach has been used in conjunction with convolutional low-level features in Section 5.2

\section{Weak labels for road mapping}

This section describes how to generate image-wide labels by matching crowd-sourced location database such as OpenStreetMap [38] to geo-referenced video.

\subsection{OpenStreetMap data representation}

The OpenStreetMap (OSM) database comprises three basic entities: nodes, ways and relations [38]. An OSM node represents a point object defined by its GPS coordinates, i.e. longitude and latitude. A way is defined as an ordered list of nodes and its purpose is to describe linear or area-like features (e.g. roads, rivers, buildings). A relation is defined as an ordered list of nodes, ways or relations and its goal is to define logical or geographical relationships (e.g. bus routes, turn restrictions or complex polygons). Each of these entities can be assigned a collection of tags which provide semantic meaning. Tags are defined as key-value pairs, where keys are used to organize entities into different categories. In general, road-environment objects are designated with the key "highway". Some examples of tags include "highway"="traffic_signals" (used to denote the traffic lights), "highway"="give_way" (used to denote the "yield" traffic sign) or "highway"="crossing" (used to denote the zebra crossing). OSM entities [39, 40] can be accessed through the Overpass website [41] which provides an interface to query the OSM database for specific features (e.g. zebra crossings, rest area facilities or traffic signs). 


\subsection{Geo-referenced video}

Geo-referenced video consists of a file with video data in one of the standard video formats, and a corresponding text file containing spatio-temporal information such as time, GPS location and camera direction. There are numerous ways on how to acquire a geo-referenced video [42] including GPS enabled cameras and camcorders, or smartphone applications like Mapillary [43] and OpenStreetView [44]. In this paper, we used geo-referenced video sequences delivered by the E-roads projects for Croatian cities of Karlovac and Sisak [27]. The obtained material consists of 1536 videos captured by different cameras, ranging from high resolution 1080p to 720p devices. Each video sequence is assigned a corresponding text file in a JSON format, where each JSON object contains an array of GPS coordinates and a time offset from the beginning of the video, e.g. \{"coordinates": [15.603174, 45.478279], "time": 53.5\}. The frequency of the GPS sampling depends on the underlying video and ranges from $5 \mathrm{~Hz}$ to $0.5 \mathrm{~Hz}$. The frame rate of video sequences corresponds to $25 \mathrm{fps}$.

\subsection{Matching OSM objects to geo-referenced videos}

We use the OSM nodes tagged as "highway"="crossing" and match them to geo-referenced video sequences to obtain positive training images with weak labels. In order to improve performance, we preprocess the list of spatiotemporal coordinates (GPS location, time offset) assigned to the video, by partitioning it into segments $\mathbf{g}$ of at least $5 \mathrm{~m}$ in length. These segments are used to quickly locate the pertinent part of the video, as illustrated in Figure 2 .

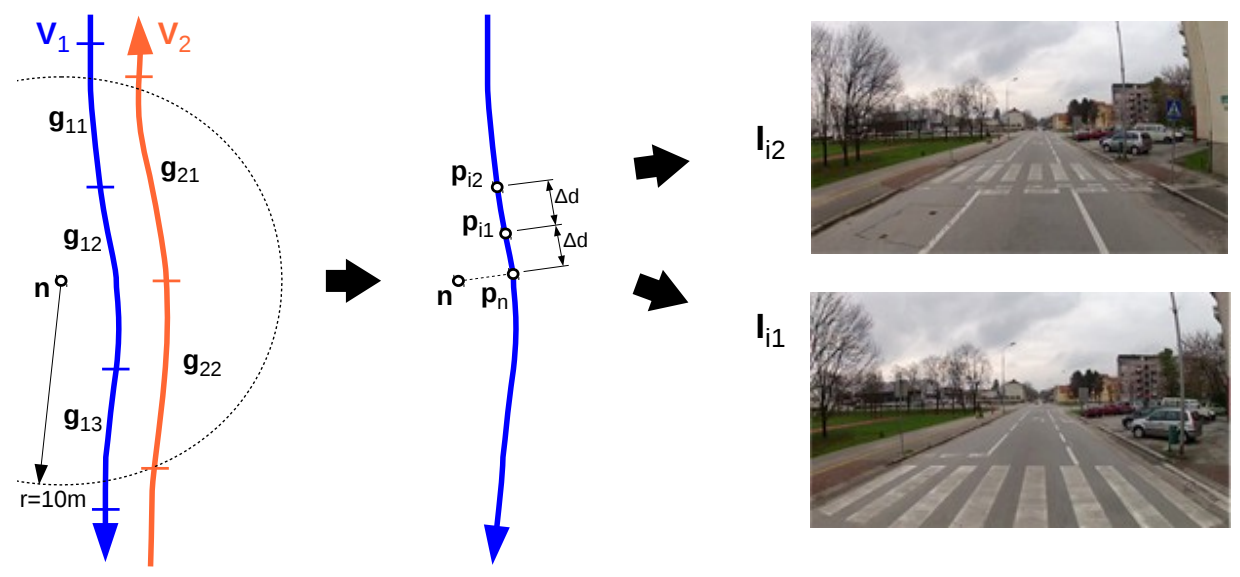

Figure 2: Matching the OSM node with osm_id $=2043645281$, located at $\mathbf{n}=45.487347 \mathrm{~N}, 15.556345$ E to geo-referenced videos $\mathbf{V}_{1}$ and $\mathbf{V}_{2}$. The first step of matching selects segments $\mathbf{g}_{\mathbf{1 2}}$ and $\mathbf{g}_{\mathbf{2 1}}$. We detail the second step for the segment $\mathbf{g}_{\mathbf{1 2}}$ : i) a GPS reference closest to $\mathbf{n}$ is located and designated as $\mathbf{p}_{n}$, ii) each of $T=2$ images is extracted by following the path backwards for $\Delta \mathrm{d}=3 \mathrm{~m}$.

For each OSM node $\mathbf{n}$, the matching algorithm considers all segments $\mathbf{g}_{i}$ which are i) in a $10 \mathrm{~m}$ radius around $\mathbf{n}$, and ii) closer to $\mathbf{n}$ than the neighbouring nodes $\mathbf{g}_{i-1}$ and $\mathbf{g}_{i+1}$. On each such segment we find the GPS reference $\mathbf{p}_{n}$ which is closest to $\mathbf{n}$. This can be seen as an approximate projection of $\mathbf{n}$ onto $\mathbf{g}_{i}$. Subsequently we take $T$ snapshots from the video every $\Delta d$ meters backward, in order to retrieve images for which the desired object is visible and close to the viewpoint. The whole procedure is summarized in Algorithm 1 


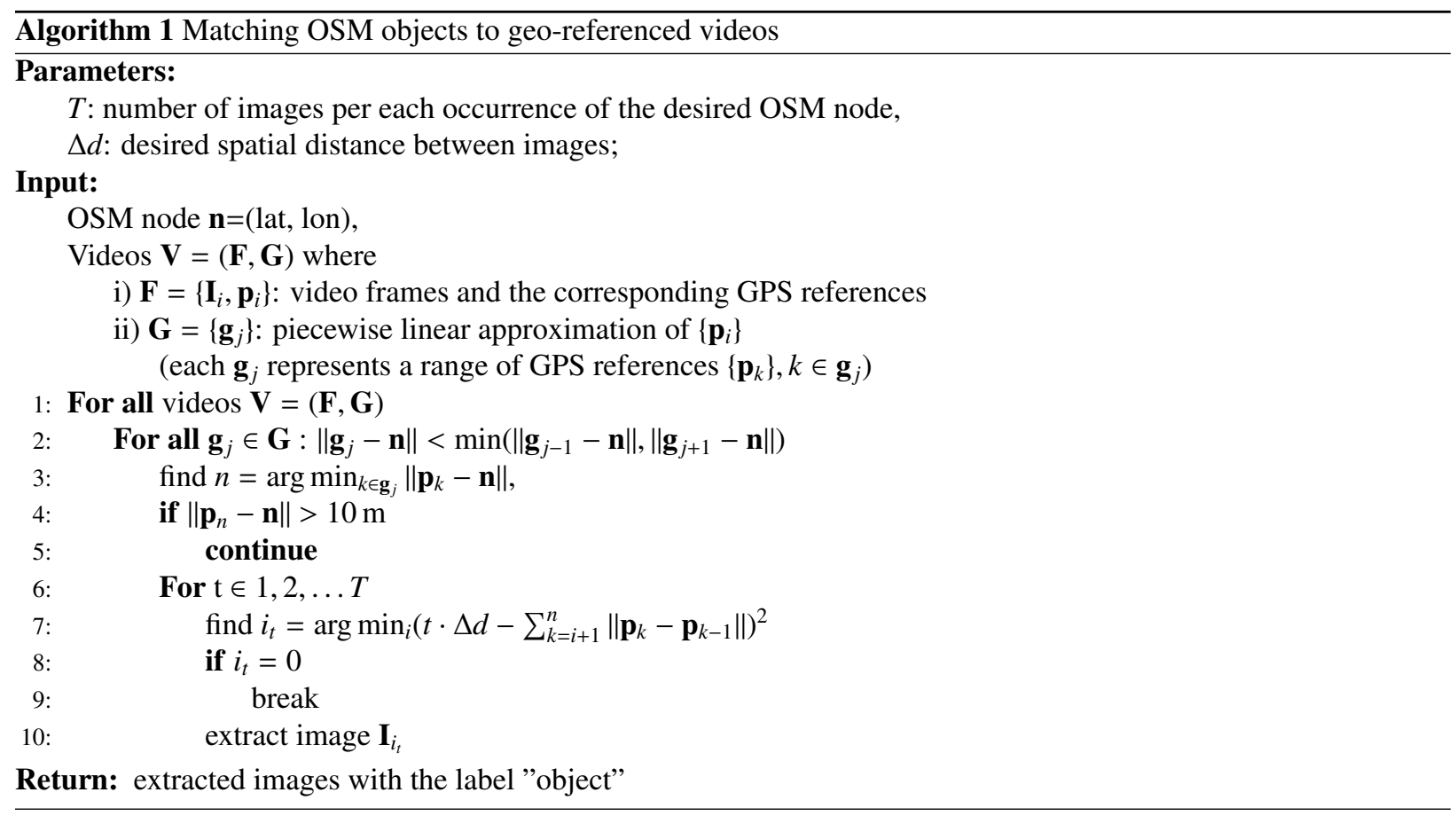

In order to account for discrepancies between the OSM geometry and GPS data, we set $T=3$ and $\Delta d=3 \mathrm{~m}$. Due to various kinds of uncertainty involved (errors in GPS references, imprecise OSM tags, etc.) the proposed algorithm may also extract some images without an object of interest. The prevalence of such images in the case of zebra crossings was around $15 \%$. In the following section we first present the results obtained by a model trained on noiseless image-wide labels, where we manually filtered out such images. We further discuss the robustness of our weakly supervised approach when training by using noisy labels (Section 5.2.4).

\section{Experimental evaluation}

We evaluate the proposed workflow on two kinds of traffic infrastructure: zebra crossings and triangular warning signs. We compare efficiency of two kinds of local features and quantify the localization accuracy with average precision (AP) [45] for various IoU thresholds. Experiments show that our method succeeds to localize objects of varying size, both very small and large, in rich traffic scenes. Besides the recognition accuracy, we also measure the execution time spent in scoring of feature embeddings as the critical part of the localization pipeline.

\subsection{Implementation details}

We perform experiments on two kinds of local features: VGG conv5_4 [18] and dense SIFT [46]. We extract approximately $80 \cdot 10^{3} 128$-D SIFT descriptors per image at 4 scales for traffic signs and 5 scales for zebra crossings. The smallest patch size was set to 16 (traffic signs) and 32 (zebra crossings) pixels, while the stride was always set to $1 / 8$ patch width. Subsequently, the descriptors are $\ell_{2}$ normalized and projected onto an $80-\mathrm{D}$ principal component subspace.

VGG conv5_4 features correspond to 512-dimensional activations from the conv5_4 layer of the deep convolutional model VGG-19 [18]. We use the provided parameters trained on ImageNet and abstain from fine-tuning [14, 10, 17] in order to avoid catastrophic forgetting [47] and preserve applicability to a diverse set of object classes. In the case of zebra crossings, we extract VGG conv5_4 features at 3 scales obtained by rescaling the image by factors $2^{s}$ where $s \in\{0,-0.5,-1\}$ [18]. In the case of traffic signs we use single scale $s=0$.

We embed the extracted local features into Fisher vectors with respect to GMMs with diagonal covariances, trained with the EM implementation from Yael [48]. We used $K_{\mathrm{SIFT}}=1024$ and $K_{\mathrm{VGG}}=128$ components. Doubling these 
figures did not produce noticeable effects on a held-out dataset. The dimensionalities of the Fisher vectors are 164864 (SIFT) and 131200 (VGG). We aggregate local Fisher vectors into image descriptors and apply different non-linear normalizations [30]. Both local and image-wide Fisher vectors are scored with linear models trained by SPAMS [49], as described in Section 3.3. The regularization hyper-parameter $\lambda$ was determined by 10 -fold cross-validation.

\subsection{Zebra crossings}

Experiments on zebra crossings provide a proof of concept for the road mapping scenario presented in Section 4. We detail the semi-automatic dataset acquisition procedure, present classification and localization experiments, and discuss the experimental results. We first present experiments on VGG conv5_4 features extracted from the dataset with noiseless labels. Experiments on SIFT descriptors and noisy labels are presented towards the end of this section.

\subsubsection{Dataset}

We collected the images from geo-referenced video obtained in the scope of a public road mapping project [27]. We recovered the locations of zebra crossings by querying the OpenStreetMap (OSM) database with the "highway=crossing" tag using the Overpass API interface [41]. We matched the object locations to geo-references of the video frames as described in Section 4. For each OSM entry, we extracted on average 6 images taken from different videos and different distances to the object, and rescaled them to the lowest common resolution $1280 \times 720$. In this way we recovered 1259 noisy positive images and 1122 negative images by random selection far from OSM entries. The extracted images depict objects under different perspectives and various weather conditions as shown in Figure 3
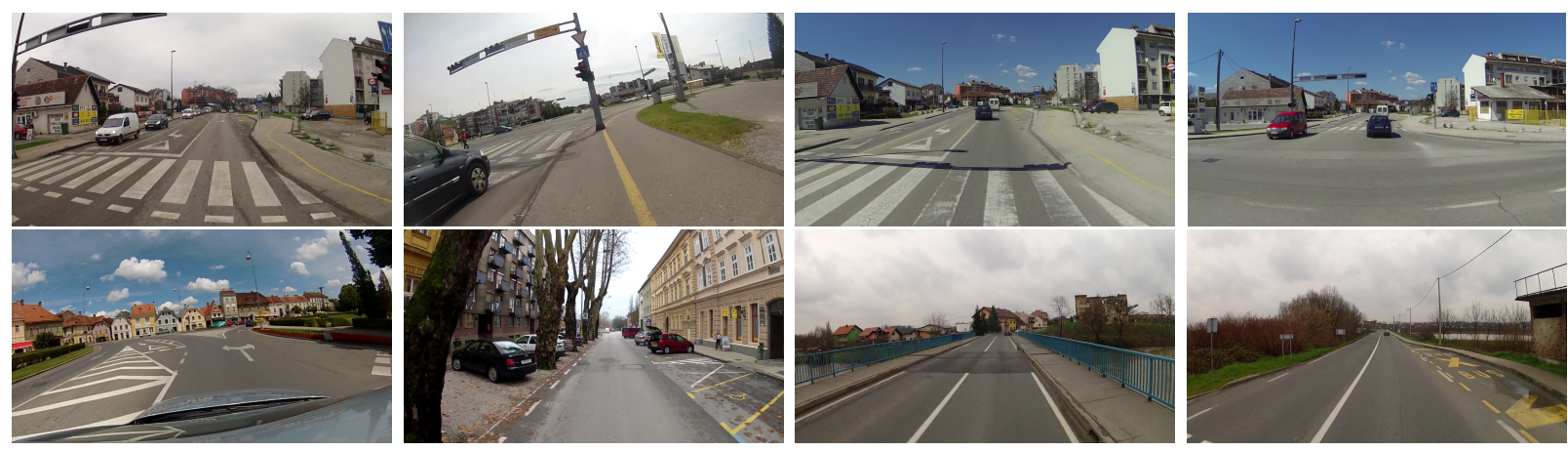

Figure 3: Top row: images extracted for the OSM node with osm_id = 981409265 located at 45.483031 N, 15.546749 E. From left to right: an image in which the zebra crossing was freshly painted, an image acquired from a bicycle, a close-up image in which the crossing was worn-out, and another image from that video taken 20 metres from the crossing. Bottom row: negative images contain many objects with patterns which are similar to zebra crossings.

We produced a clean version of the dataset by manual filtering of the 1259 noisy positives into 1067 positives and 192 negatives, and by manual verification that the 1122 negatives do not contain an unmapped object. The cleaning procedure took around 20 minutes. We split both versions of the dataset into roughly equal train and test subsets by taking care that all physical objects are assigned to the same subset.

In order to evaluate the localization performance, we annotated the ground-truth object locations in the test set with polygonal approximations. Some of the test images contain several objects at different distances from the camera. For the sake of completeness, we annotated all of them, even the smallest ones. Details concerning the statistics of annotated objects with respect to the image area are shown in Table 1. Hence, from 484 positive test images we obtained 674 object annotations, where 337 images contain a single object, 120 of them contain 2 objects, 14 contain 3 objects and another 13 contain more than 3 objects. The complete dataset shall be made public upon publication of this manuscript.

\subsubsection{Classification with VGG conv5_4 and noiseless labels}

These experiments evaluate effects of cross-validated regularization and non-linear normalization to the classification AP. The first section of Table 2(rows \#1-\#3) assumes that images are represented with raw Fisher vectors. We 
Table 1: Annotated object size statistics for the zebra crossing dataset. The first column contains the object size intervals expressed in percentages of an image area. The second column indicates the overall share of objects in the corresponding size interval.

\begin{tabular}{cc}
\hline Relative object size & Frequency \\
\hline$<1 \%$ & $30 \%$ \\
$2 \%-7 \%$ & $48 \%$ \\
$>7 \%$ & $22 \%$ \\
\hline
\end{tabular}

notice that the $\ell_{2,1}$ regularization succeeds to induce a five-fold increase of component-level sparsity with respect to $\ell_{1}$ regularization, which shall be especially useful in localization experiments.

Table 2: Classification of zebra crossings with different FV normalizations (p: power, $\ell_{2}$ global, $\ell_{2}$ intra) and regularizations $\left(\ell_{1}, \ell_{2}, \ell_{2,1}: \ell_{2}\right.$ inside component, $\ell_{1}$ between components). Average overall density (AOD) and average component density (ACD) denote percentages of non-zero model coefficients and non-zero model coefficient groups, respectively.

\begin{tabular}{cccrrrc}
\hline Nr. & FV norm. & Penalty & AOD & ACD & AP train & AP test \\
\hline 1 & - & $\ell_{2}$ & 77.4 & 100.0 & 97 & 95 \\
2 & - & $\ell_{1}$ & 2.1 & 78.9 & 94 & 95 \\
3 & - & $\ell_{2,1}$ & 8.5 & 14.8 & 95 & 95 \\
\hline 4 & $\mathrm{p}, \ell_{2}$ global & $\ell_{2}$ & 77.4 & 100.0 & 100 & 97 \\
5 & $\mathrm{p}, \ell_{2}$ global & $\ell_{1}$ & 0.2 & 30.5 & 100 & 98 \\
6 & $\mathrm{p}, \ell_{2}$ global & $\ell_{2,1}$ & 3.8 & $\mathbf{7 . 0}$ & 100 & 98 \\
\hline 7 & $\mathrm{p}, \ell_{2}$ intra & $\ell_{2}$ & 77.4 & 100.0 & 100 & 97 \\
8 & $\mathrm{p}, \ell_{2}$ intra & $\ell_{1}$ & 0.1 & 21.1 & 97 & 98 \\
9 & $\mathrm{p}, \ell_{2}$ intra & $\ell_{2,1}$ & 2.6 & $\mathbf{4 . 7}$ & 100 & 98 \\
\hline
\end{tabular}

The next section (rows \#4-\#6) considers models trained on Fisher vectors with power and global $\ell_{2}$ normalization. We notice that non-linear normalizations increase the classification performance (all models) and the component-level sparsity $\left(\ell_{1}\right.$ and $\left.\ell_{2,1}\right)$. We believe that non-linear normalizations promote the model sparsity by decreasing the range of Fisher vector elements and therefore encouraging the model to bring decisions by testing presence instead of testing the aggregated value. Finally, we consider intra-component $\ell_{2}$ normalization (rows \#7-\#9). This technique works especially well in conjunction with the group-sparse $\ell_{2,1}$ regularization (row \#9), since the combined model retained only $5 \%$ of GMM components while reaching the same level of classification performance as in the case of global $\ell_{2}$ normalization (rows \#4-\#6).

To summarize, the sparse models achieve comparable or better results with respect to their dense counterparts, despite the fact that they utilize only a tiny fraction of the visual dictionary. Non-linear normalizations boost the performance on the test dataset up to 3 percentage points (pp).

\subsubsection{Localization with VGG conv5_4 and noiseless labels}

In these experiments, we train the model on entire images, apply it to all image patches at multiple scales, and recover objects as described in Section 3 . In order to compensate for large variability in scale and semi-automatic dataset generation, we first use a permissive IoU threshold [45] of 0.10 while other IoU thresholds shall be considered in one of the following paragraphs. The obtained results are summarized in Table 3 and Figure 4.

The first section of Table 3 (rows \#1-\#3) shows that sparse models outperform the dense baseline $\left(\ell_{2}\right)$ for up to $16 \mathrm{pp}$ in AP and $15 \mathrm{pp}$ in $p_{\text {miss }}$. We also note that the $\ell_{2,1}$ model (row \#3 in Table 3 ) outperformed the $\ell_{1}$ model (row \#2), despite employing much less GMM components. The first row in Figure 4 reflects that result: the $\ell_{2,1}$ model (far right) selects less background pixels than the $\ell_{1}$ and $\ell_{2}$ models. This advantage was consistent in all our experiments and supports the hypothesis outlined in Section 3.3 that the $\ell_{2,1}$ model may perform better due to agreement with the group structure of the Fisher vector. Hence, we include only the results of $\ell_{2,1}$ models in the rest of the table. The middle section of Table 3 (rows \#4-\#5) employs normalized Fisher vectors and computes the patch contribution 
Table 3: Localization of zebra crossings with different configurations (M: direct patch contribution, G: gradient) and FV normalizations. $p_{\text {miss }}$ denotes the miss frequency at the rightmost data point of the PR curve. $t_{\mathrm{op}}$ denotes the average time required to compute the scores for the selected patches. For gradient configurations (rows \#6, \#7), we show the speed-up w.r.t. corresponding row with direct patch contribution (rows \#4, \#5).

\begin{tabular}{rcccrrrrr}
\hline Nr. & Conf. & FV norm. & Penalty & ACD & AP test & $p_{\text {miss }}$ & $t_{\text {op }} / \mathrm{s}$ & speed-up \\
\hline 1 & $\mathrm{M}$ & - & $\ell_{2}$ & 100.0 & 76 & 0.46 & 26.6 & \\
2 & $\mathrm{M}$ & - & $\ell_{1}$ & 100.0 & 91 & 0.33 & 19.1 & \\
3 & $\mathrm{M}$ & - & $\ell_{2,1}$ & 14.8 & 92 & 0.31 & 2.8 & \\
\hline 4 & $\mathrm{M}$ & $\mathrm{p}, \ell_{2}$ global & $\ell_{2,1}$ & 7.0 & 92 & 0.27 & 29.8 & \\
5 & $\mathrm{M}$ & $\mathrm{p}, \ell_{2}$ intra & $\ell_{2,1}$ & 4.7 & $\mathbf{9 3}$ & $\mathbf{0 . 2 5}$ & 0.8 & \\
\hline 6 & $\mathrm{G}$ & $\mathrm{p}, \ell_{2}$ global & $\ell_{2,1}$ & 7.0 & 90 & 0.28 & 1.0 & $28.8 \times$ \\
7 & $\mathrm{G}$ & $\mathrm{p}, \ell_{2}$ intra & $\ell_{2,1}$ & 4.7 & $\mathbf{9 2}$ & $\mathbf{0 . 2 5}$ & $\mathbf{0 . 3}$ & $2.7 \times$ \\
\hline
\end{tabular}

directly as $f(\mathbf{X})-f(\mathbf{X}-\mathbf{x})$. This further decreases the $p_{\text {miss }}$ frequency and selects fewer pixels in the background as confirmed by rows 2 and 3 in Figure 4 . We notice that the best localization result (93\% AP and $0.25 p_{\text {miss }}$ ) is obtained by intra-component $\ell_{2}$ normalization which constrains the influence of unusual patches to the components with significant soft-assign probability. We also note that intra-component $\ell_{2}$ normalization has a negative effect when $\ell_{1}$ regularization is used. We believe this happens because $\ell_{1}$ regularization disrupts the component structure of the Fisher vector and thus makes intra-component normalization counter-effective for localization purposes. The final section of Table 3 (rows \#6-\#7) evaluates the effects of the gradient approximation (9) to the configurations from the previous section (rows \#4-\#5). The results show that the performance hit is minimal: up to 2 pp drop in AP, and up to $1 \mathrm{pp}$ increase in $\mathrm{p}_{\mathrm{miss}}$. We arrive to the same conclusion by performing a qualitative comparison between rows $2-3$, and rows 4-5 in Figure 4

Failure cases. Figure 5 shows representative examples of our best configuration (row \#5 in Table 3 ). The correct localization polygons are shown in yellow, incorrect ones are shown in red while ground truth polygons for the missing localizations are shown in magenta. Our method is able to localize objects taken from different viewpoints (frontal, lateral) as well as worn-out objects (cf. row 1, far right). Most false negatives (80\%) correspond to extremely small objects far away from the viewpoint. The remaining false negatives arise in images with adjacent objects (ground-truth polygons are touching each other) where our method reports one instead of two or more objects (cf. rows 2 and 3 , far right). The average size of the missed objects corresponding to the first failure type is $4573 \mathrm{px}$, which is only $0.5 \%$ of the total image. As we already pointed out in Section 3, such failures are not critical for accurate road mapping where we care most about the objects which are close to the viewpoint.

IoU threshold. Figure 6 explores the influence of the IoU threshold to the localization performance for our best configuration (row \#5 in Table 3). The left subfigure shows the counts of objects and the corresponding average IoU depending on the ground-truth size interval. The graph reveals that most of objects which are missed for IoU $<0.1$ are smaller than $10^{4}$ pixels which corresponds to $1 \%$ of the image size. The right subfigure shows the effect of the IoU threshold to average precision and the miss frequency for objects which are greater than $10^{4}$ pixels. The graph shows that the increase of IoU threshold results in gradual decrease of AP and increase in $\mathrm{p}_{\text {miss. }}$ Setting IoU to 0.3 results in $80 \% \mathrm{AP}$ and $0.3 p_{\text {miss }}$, which is still a fairly good localization accuracy in a weakly supervised setting. Further increase of the IoU threshold severely degrades the AP due to false negatives and failures to distinguish nearby objects.

Execution time. Finally, we provide a brief analysis of the execution profile. We extract on average $6344 \mathrm{CNN}$ features per image, which takes around $t_{\mathrm{lf}}=1 \mathrm{~s}$ on an NVidia GTX 980 GPU. All subsequent processing is performed on a single CPU core of a $2.00 \mathrm{GHz}$ Intel Xeon E5-2620. Computing the soft assign probability for the extracted features takes $t_{\mathrm{sa}}=0.11 \mathrm{~s}$. The times $t_{\mathrm{lf}}$ and $t_{\mathrm{sa}}$ are constant for all configurations. Our Python implementation takes on average $1.9 \mathrm{~s}$ per image $\left(t_{\mathrm{lf}}+t_{\mathrm{sa}}+t_{\mathrm{op}}\right)$ for our best configuration in terms of AP (row \#5 in Table 3 ). The gradient approximation further reduces that time to $1.4 \mathrm{~s}$ with a small penalty on the localization AP (row \#7 in Table 3 ).

We conclude that $\ell_{2,1}$ models are faster than their $\ell_{1}$ counterparts due to a better chance of having an empty intersection with the Fisher vector of image patches (improvement over $\ell_{2}$ models is even larger). Intra-component 
normalization further decreases the processing time since it allows to pre-compute most components of $f(\mathbf{X}-\mathbf{x})$ when patch contributions are computed directly. Finally, the gradient approximation allows to score a patch with a simple dot product. Gradient approximation works very well with intra-component normalization since the latter preserves the sparsity of $\partial f(\mathbf{X}) / \partial x_{d}$ and therefore leads to empty intersections for most patches. When compared with the baseline (row \#1 in Table 3), our fastest configuration (row \#7 in Table 3 ) leads to 89-fold improvement in $t_{\text {op }}$ or about 20 -fold improvement in overall execution time.

\subsubsection{Comparison with SIFT and noisy labels}

Table 4 further explores our best configuration in terms of localization AP by presenting results with different features and noisy labels. For convenience of comparison, the row \#1 repeats the results from the row \#5 in Table 3 . The row \#2 shows effects of replacing VGG conv5_4 features with dense SIFT features. The results show that our
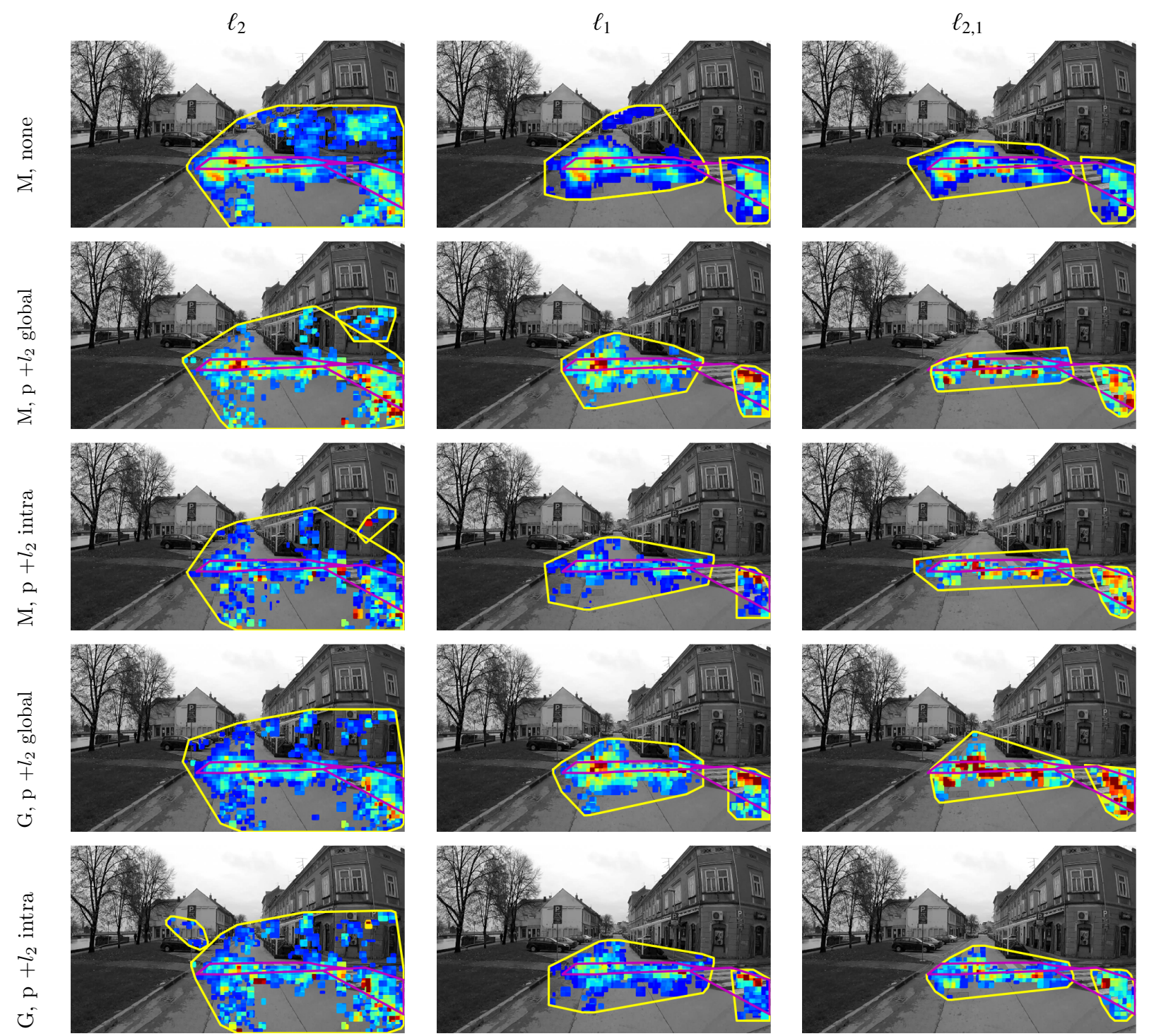

Figure 4: A comparison of multi-scale heat maps for different non-linear normalizations (rows) and different regularizations (columns). The input image contains 2 zebra crossings: one in the lateral position very close to the viewpoint, and another one in the frontal position 10 meters from the camera. Magenta polygons denote the ground truth instances; they are used for evaluation purpose only. Yellow polygons denote the resulting localization responses. 

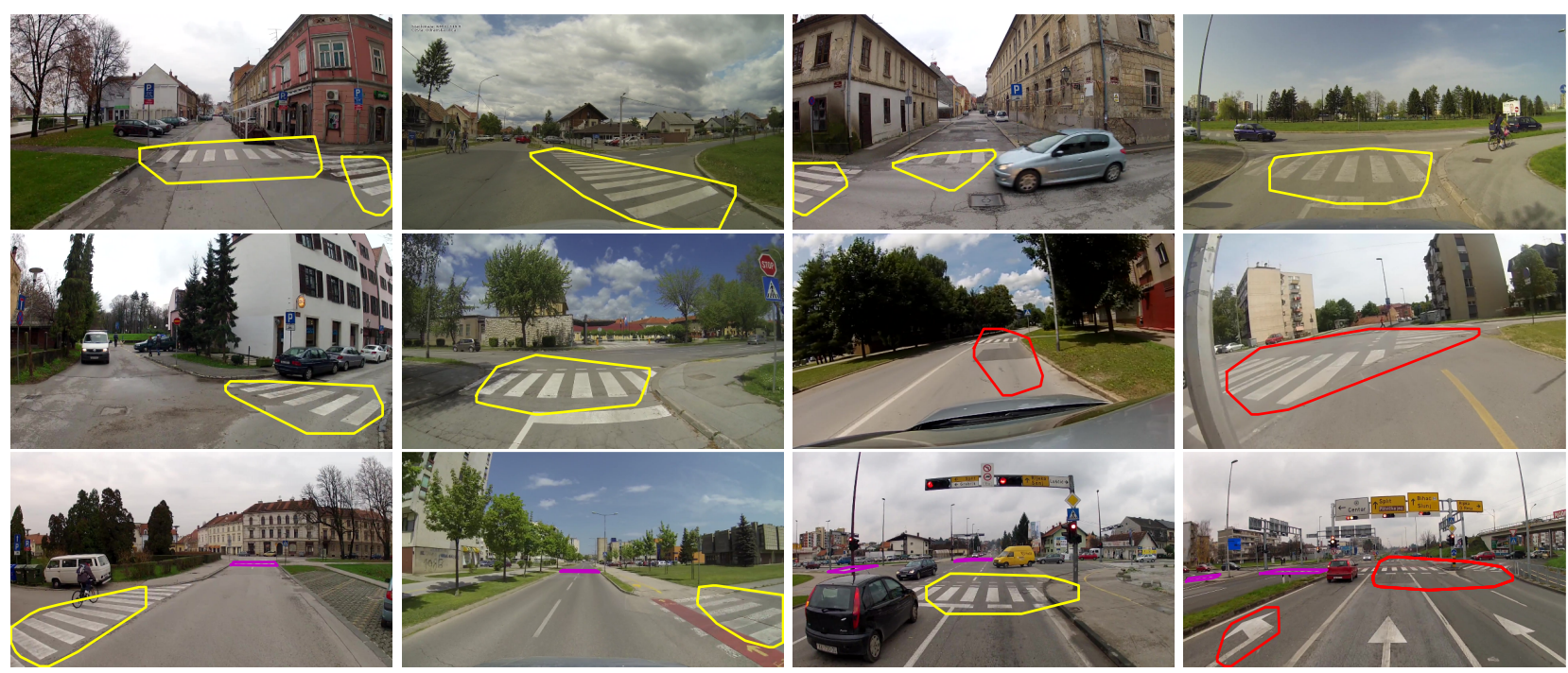

Figure 5: Localization results on test images: correct localization polygons (yellow), false postive responses (red), and ground-truth polygons for false negatives (magenta).

weakly supervised pipeline is able to successfully accommodate different kinds of local features on this dataset. The row \#3 shows effects of training the model on raw image dataset obtained by sampling the geo-referenced video at GPS locations provided by crowdsourced OSM tags. The raw dataset contained significant noise in positive labels since around each 9th positive image in the training set was in fact a negative (we used a clean test dataset in all experiments). Nevertheless the obtained results show that this leads to only a slight decrease (1 pp) in localization AP. We hypothesize that this robustness is due to regularization imposed by the $\ell_{2,1}$ penalty and the low capacity of the chosen classification model. This suggests that fully automated road environment mapping is feasible.

Table 4: Comparison of our best configuration in terms of localization AP (row \#5 in Table 3 ) with the results obtained with different features and noisy labels. We used $\ell_{2,1}$ regularization and direct computation of the patch contribution in all experiments. In experiments on VGG conv5_4 we used intra-component normalization, while in experiments on traffic signs we used raw Fisher vectors.

\begin{tabular}{cllrrrr}
\hline Nr. & Features & Training set & ACD & AP test & $p_{\text {miss }}$ & $t_{\text {op }} / \mathrm{s}$ \\
\hline 1 & VGG conv5_4 & noiseless & 4.7 & 93 & $\mathbf{0 . 2 5}$ & 0.8 \\
2 & SIFT & noiseless & 4.1 & $\mathbf{9 5}$ & 0.26 & 0.9 \\
3 & VGG conv5_4 & noisy & 3.9 & 92 & 0.25 & 0.7 \\
\hline
\end{tabular}

\subsection{Traffic signs}

Experiments on traffic signs explore the capability of our approach to deal with extremely small objects. We focus on 33 different kinds of European triangular warning signs [50] which we treat as a single object class in weakly supervised localization experiments. We first present experiments on SIFT features, while the comparison with VGG conv5_4 is presented in the subsequent subsection.

\subsubsection{Dataset}

We use a public traffic sign dataset [51] and adapt it for weakly supervised localization? The adapted dataset contains 1705 train and 1591 test images. Most images contain only one traffic sign while around 16\% images contain

\footnotetext{
${ }^{2}$ The adapted traffic sign dataset can be downloaded from http://multiclod.zemris.fer.hr/ts2010a.shtml
} 

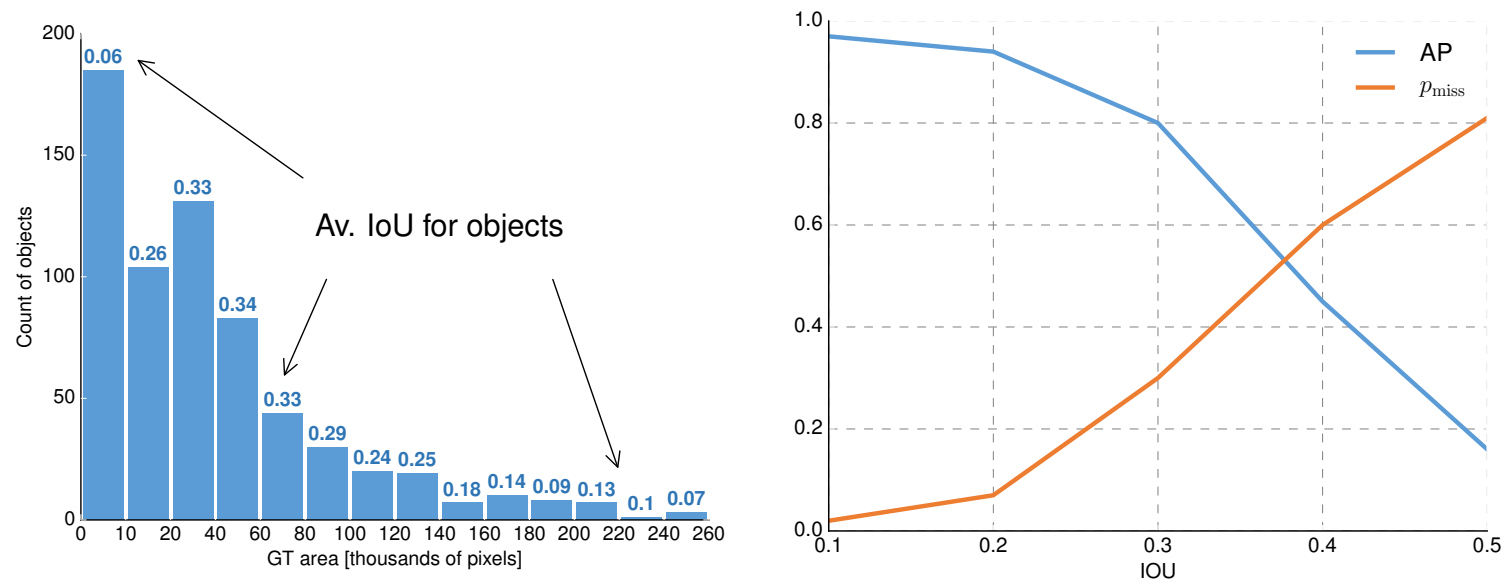

Figure 6: Influence of the IoU threshold to the localization precision on the zebra crossings dataset for the configuration \#5 from Table 3 Left: distribution of objects over a range of ground-truth area intervals. For each ground-truth area range, we report the average IoU. Right: the effect of the IoU threshold on AP and $p_{\text {miss }}$ for objects greater than $1 \%$ of the image area.

two traffic signs. The train and test subsets are disjoint: all physical object are present in only one subset. The main challenge in this dataset is object size, since many instances are less than $30 \times 30$ pixels large $(0.2 \%$ of image size $)$. All images are correctly labeled and they have the common resolution $720 \times 576$.

\subsubsection{Classification with SIFT features}

Classification experiments evaluate effects of cross-validated regularization and non-linear normalization. The results are summarized in Table 5. Group sparse models $\left(\ell_{2,1}\right)$ outperform alternatives $\left(\ell_{1}\right.$ and $\left.\ell_{2}\right)$ in all configurations, both with respect to generalization performance (AP test) and average component density (ACD). This can be observed in rows \#1-\#3 which address classification with unnormalized Fisher vectors. Similar effects occur with normalized

Table 5: Image classification performance for traffic signs with different FV normalizations (p: power, $\ell_{2}$ global, $\ell_{2}$ intra) and regularizations $\left(\ell_{1}\right.$, $\ell_{2}, \ell_{2,1}: \ell_{2}$ inside component, $\ell_{1}$ between components). AOD (average overall density) denotes the percentage of non zero model coefficients (out of 164865 total). ACD (average component density) denotes the percentage of non-zero model components (out of 1024 total).

\begin{tabular}{cllrrrc}
\hline Nr. & FV normalization & Penalty & AOD & ACD & AP train & AP test \\
\hline 1 & - & $\ell_{2}$ & 92.8 & 100.0 & 100 & 66 \\
2 & - & $\ell_{1}$ & 0.1 & 6.1 & 87 & 71 \\
3 & - & $\ell_{2,1}$ & 1.0 & 1.1 & 83 & 75 \\
\hline 4 & p, $\ell_{2}$ global & $\ell_{2,1}$ & 1.1 & 1.1 & 87 & $\mathbf{8 1}$ \\
5 & p,$\ell_{2}$ intra & $\ell_{2,1}$ & 0.8 & 0.8 & 85 & 80 \\
\hline
\end{tabular}

Fisher vectors as well, however in that case we only show the group sparse models in order to save space (rows \#4-\#5). We achieve the best classification performance with global normalization (row \#4, 81\% AP), however, intracomponent normalization is only marginally worse (row \#5, 80\% AP).

\subsubsection{Localization with SIFT features}

We follow the same experimental setup as in the case of zebra crossings. Table 6 shows the obtained results. Due to the fact that the traffic sign dataset [51] contains ground truth bounding boxes for both train and test splits, we were able to train a strongly supervised baseline [52] (row \#1). We employ the standard OpenCV implementation and invoke it through Python interface cv2. HOGDescriptor. detectMultiScale. We configure the baseline approach for high accuracy, by adjusting parameters of the sliding window according to the range of scales at which the traffic signs appear in our dataset. Thus we extract the HOG features at 64 different scales ranging from $24 \times 24$ to $160 \times 160$ 
(the inter-scale factor was set to 1.03), while the stride was set to two pixels. In comparison to our best weakly supervised result (row \#5: 92\% AP, $0.15 p_{\text {miss }}$ ), the strongly supervised baseline achieves worse AP (88\%) and better miss frequency (only $5 \%$ of objects were not found). However, please note that we extract local features on only 4 scales, which is 8 times less than the baseline (64 scales).

Table 6: Localization performance for traffic signs. Configuration B denotes strongly supervised baseline (HOG [52]); the respective execution time includes both $t_{\mathrm{op}}$ and $t_{\mathrm{lf}}$. The remaining notation is the same as in previous tables.

\begin{tabular}{llllrrrrr}
\hline Nr. & Conf. & FV Norm. & Penalty & ACD & AP test & $p_{\text {miss }}$ & $t_{\text {op }} / \mathrm{s}$ & speed-up \\
\hline 1 & B & - & $l_{2}$ & - & 88 & $\mathbf{0 . 0 5}$ & 10.0 & \\
\hline 2 & M & - & $l_{2}$ & 100.0 & 66 & 0.27 & 8.9 & \\
3 & M & - & $l_{1}$ & 6.1 & 81 & 0.21 & 1.9 & \\
4 & M & - & $\ell_{2,1}$ & 1.1 & 84 & 0.23 & 0.1 & \\
\hline 5 & M & p,$\ell_{2}$ global & $\ell_{2,1}$ & 1.1 & $\mathbf{9 2}$ & 0.15 & 27.1 & \\
6 & M & p,$\ell_{2}$ intra & $\ell_{2,1}$ & 0.8 & 88 & 0.13 & 0.3 & \\
\hline 7 & G & p,$\ell_{2}$ global & $\ell_{2,1}$ & 1.1 & 87 & 0.15 & $\mathbf{0 . 1}$ & $226.0 \times$ \\
8 & G & p,$\ell_{2}$ intra & $\ell_{2,1}$ & 0.8 & 86 & $\mathbf{0 . 1 2}$ & $\mathbf{0 . 1}$ & $4.5 \times$ \\
\hline
\end{tabular}

Experiments with weakly supervised models support our hypotheses (i) that sparse models are a good match for weakly supervised localization, and (ii) that gradient approximation retains localization accuracy and gains execution speed. The second section (rows \#2-\#4) shows that the sparse models $\left(\ell_{1}, \ell_{2,1}\right)$ substantially outperform the dense baseline $\left(\ell_{2}\right)$. Recognition performance is further improved by non-linear normalizations (rows \#5-\#6). Similarly to zebra crossings, intra-component $\ell_{2}$ normalization yields a lower $p_{\text {miss }}$. Contrary to zebra crossings and similarly to classification results, intra-component $\ell_{2}$ normalization yields a lower AP. Experiments with the gradient approximation (rows \#7-\#8) achieve slightly worse AP than direct patch contribution (rows \#5-\#6). Nevertheless, gradient approximation of intra-component normalization (row \#8) yields the best $p_{\text {miss }}$ performance in all weakly supervised experiments.

Failure cases. Figure 7 shows the results of our best configuration (row \#5 in Table 6). The first row shows that our method is able to successfully localize very small objects in both urban and rural traffic scenes. The second row shows two types of failure cases: (i) false positives appearing as parts of house roofs or other traffic signs seen from behind (the first two images in the second row), (ii) cases where top rated patches are located on objects, but either the patch connectivity is insufficient, or there are too few patches to form a bounding box. These problems could be mitigated by exploiting the spatial relationship between the visual words to rule out patches in the background [16].

Execution time. As in the case of zebra crossings, we perform all experiments on a single core of a $2.00 \mathrm{GHz}$ Intel Xeon E5-2620 CPU. We extract on average $87 \cdot 10^{3}$ patches from the input image, which takes $t_{\mathrm{lf}}=0.7 \mathrm{~s}$. The soft-assign step takes approximately $t_{\mathrm{sa}}=3.7 \mathrm{~s}$, which is a 33-fold increase with respect to the setup used for zebra crossings. This increase is a combined effect of (i) a 14-fold increase in the number of features, (ii) an 8-fold increase in the number of components (1024 vs 128) and (iii) a 6-fold decrease in feature dimensionality (80 vs 512 ). As in the case of zebra crossings, group sparsity and gradient approximation substantially decrease the execution time. Processing an image with the configuration \#8 (cf. Table 6) takes on average $4.4 \mathrm{~s}$ which is more than two times faster than the strongly supervised HOG approach.

\subsection{Comparison with related work}

We compare our best configurations from Tables 3, 4] and 6 with the related approach [13] which is similar to our work since it also refrains from fine-tuning pre-trained convolutional features. However, instead of Fisher embedding and linear classification, they postprocess convolutional features with a mini-net comprised of two adaptation layers which are subsequently pooled and trained with the maximum likelihood classification loss. The adaptation layers are implemented as convolutions $3 \times 3 \times \mathrm{D} \times 2048$ and $3 \times 3 \times 2048 \times \mathrm{C}$, where $\mathrm{D}$ is dimensionality of input features, while $\mathrm{C}$ is the number of classes. We have obtained the best results by performing the following changes to the original setup 

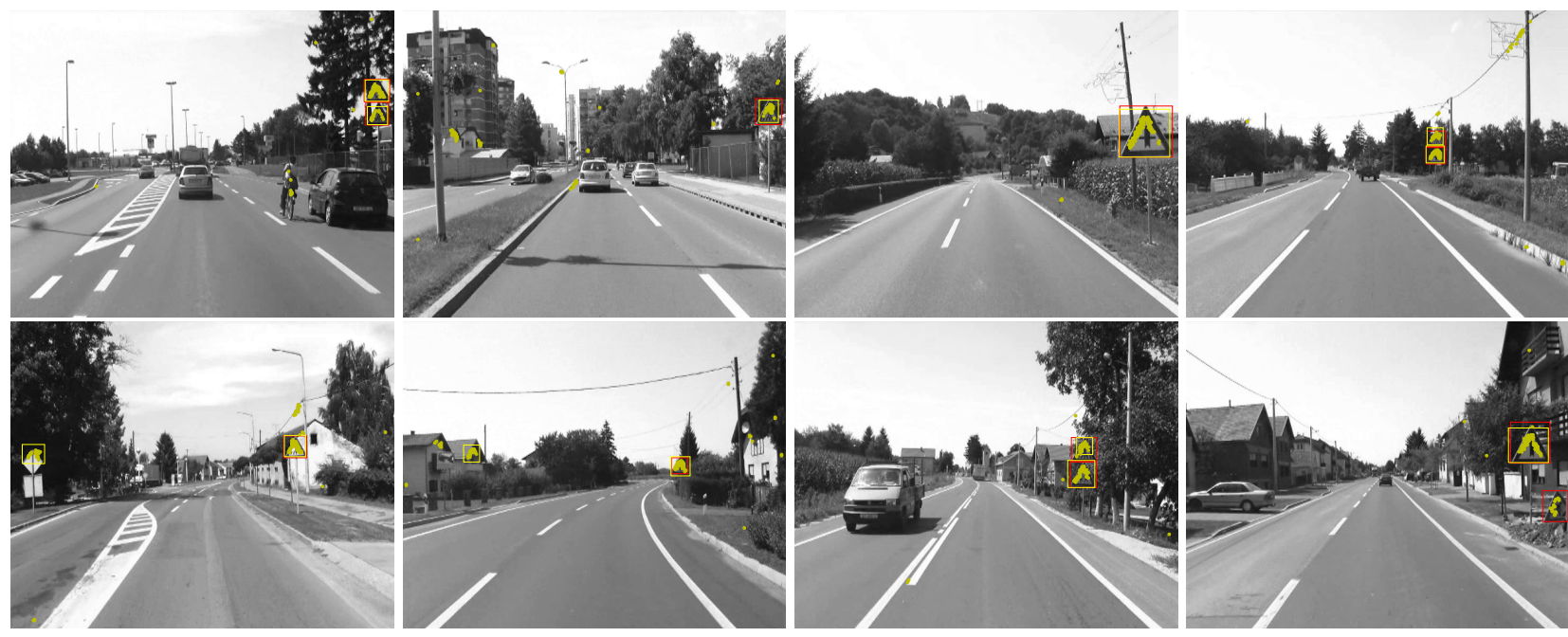

Figure 7: Examples of localization results for traffic signs: the top row shows correct localizations, the bottom one shows examples of failure cases. Localization responses are shown as yellow rectangles, the ground truth annotations (used for evaluation purpose only) are shown as red rectangles, centers of top rated patches are illustrated as yellow dots. The images are shown using grayscale colormap to emphasize the locations of top rated patches.

[13]: i) we replaced the global max pool [13] with global average pool [14], ii) we trained with dropout regularization in both adaptation layers, iii) for fairness, we use the same convolutional front-end as in our experiments (VGG conv5_4). The results are summarized in Table 7

Table 7: Comparison of our best localization results with the related approach [13] on both datasets. We repeat the best results from Table 3 Table 4 and Table 6 and provide additional experimental results on traffic signs with VGG conv5_4 features (row \#4).

\begin{tabular}{ccclccc}
\hline Nr. & Dataset & IoU & Features & Aggreg. \& scoring & AP test & $p_{\text {miss }}$ \\
\hline 1 & zebras & 0.1 & VGG conv5_4 & FV + linear & 93 & $\mathbf{0 . 2 5}$ \\
2 & zebras & 0.1 & SIFT & FV + linear & $\mathbf{9 5}$ & 0.26 \\
3 & zebras & 0.1 & VGG conv5_4 & mini-net [13] & $\mathbf{9 5}$ & 0.32 \\
\hline 4 & signs & 0.5 & VGG conv5_4 & FV + linear & 39 & 0.72 \\
5 & signs & 0.5 & SIFT & FV + linear & $\mathbf{9 2}$ & $\mathbf{0 . 1 5}$ \\
6 & signs & 0.5 & VGG conv5_4 & mini-net [13] & 17 & 0.89 \\
\hline
\end{tabular}

The first section (rows \#1-\#3) contains experiments on zebra crossings (IoU=0.1) while the second section (rows \#4-\#6) presents experiments on traffic signs (IoU=0.5). Our approach produced better localization results on both datasets. On the traffic sign dataset we obtain both higher AP and lower $p_{\text {miss }}$ as shown in rows 4 and 6 . On the zebra dataset, we achieve comparable AP and lower miss frequency (rows 1 and 3). The results on traffic signs also show that convolutional features pre-trained on ImageNet are unable to locate very small objects such as traffic signs. These results would improve after fine-tuning, however that might adversely affect the scalability of the approach and simplicity of training. The results on both datasets show that SIFT features are able to outperform pre-trained convolutional features in the cases of very small objects and objects with simple structure.

Finally, we present weakly supervised localization experiments on Pascal VOC 2012. As we shall see, this dataset is not very well-suited for our method due to strong classification cues provided by the context. Nevertheless, we present these results in order to enable comparison with other methods on this widely used dataset. As in Table 7 . we compare our method with the mininet comprised of two $3 \times 3$ adaptation layers initialized from scratch [13]. The mininet is applied to single-scale VGG conv5_4 features, and trained with dropout and average pooling. As in Table 7 , the max pooling variant resulted in very poor generalization. Log-mean-exp pooling was better than max-pooling, but 
still worse than average pooling which we used in the end. We believe these difficulties are due to different features [13] and much less training data than in [14]. Our method embeds single-scale VGG conv5_4 features into a Fisher space according to a GMM with 64 components (doubling this led to same results). We employ the best configuration from Table 3 . power and intra-component normalization and cross-validated $\ell_{2,1}$ regularization. Our experiments measure the localization AP for the strongest per-class response [13]. The results are summarized in Table 8

Table 8: Comparison of our method (FV) with the mininet approach [13] (MN) on Pascal 2012 val. We report classification (C) and localization performance (L). The localization performance considers only the strongest per-class response [13]. Both approaches operate on VGG conv5_4 features. In order to account for context, we postprocess our per-class responses by 6 convolutions with the $3 \times 3$ mean filter.

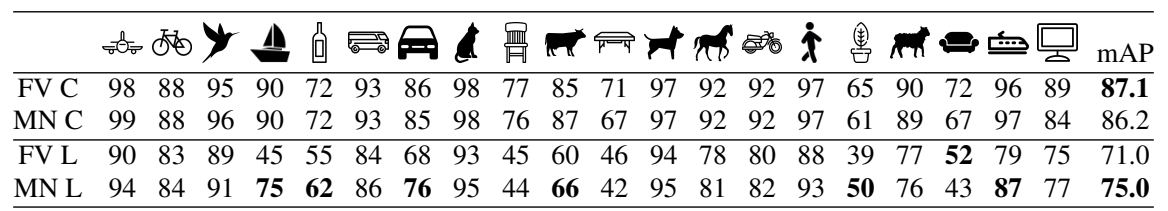

Classification results are presented in the first two rows of the table where we see that Fisher vectors outperform mininet for $0.9 \mathrm{pp}$. The employed linear model has less parameters than the mininet approach (131201 vs more than nine million) and is therefore less likely to overfit. Unfortunately, most of our models were dense (ACD $>50 \%)$, which makes them unsuitable for localization. This is reflected in the localization results where mininet outperforms our approach for $4 \mathrm{pp}$ with the exception of the sofa class (there we got a semi-dense model with ACD $=50 \%$ ). These results suggest that our method is suitable for datasets such as those from our main experiments, where the object class cannot be inferred from context.

\subsection{Discussion}

We have evaluated the performance of our method on two different road-driving datasets. In both cases, the experiments point out four facts: i) sparse models achieve better localization accuracy with faster run-time, ii) nonlinear normalizations increase the localization performance of sparse models, iii) the gradient approximation achieves comparable localization accuracy while substantially improving the run-time, and iv) intra-component normalization increases the sparsity and improves $p_{\text {miss }}$ when used in conjunction with group-sparse regularization while otherwise achieving negative effects.

We also note certain differences between the two datasets. For the classification task, the sparse models outperform the dense models on traffic signs (up to $9 \mathrm{pp}$ ), but achieve comparable performance on zebra crossings. For the localization task, the sparse models achieve better performance on both datasets. In the case of zebra crossings, there are other objects which commonly appear in positive images (e.g. traffic signs, traffic lights, or road surface markings). Hence, dense models are able to compensate the loss of focus by relying on context. Classification performance of zebra crossings is much better than for traffic signs (97\% vs 81\%). This performance gap can be explained as follows: (i) zebra crossings are in general larger than traffic signs and as such produce a larger contribution to image representation, (ii) other objects co-occurring with zebra crossings in positive images provide additional classification evidence. Clearly, these co-occurring distractors make weakly supervised localization harder. Fortunately, their impact can be alleviated with sparse models. Results from Table 3 and Figure 4 show that sparse models are able to identify patches on zebra crossings as the ones responsible for the image label, while dense models are not able to ignore other content characteristic for positive images. Finally, nonlinear normalizations improve both the localization AP and $p_{\text {miss }}$ on traffic signs, while on zebra crossings they yield comparable AP and only decrease the miss frequency.

The overall execution time for zebra crossings is 3 times faster than for traffic signs. This discrepancy is due to soft-assign stage being 33 times slower in the case of traffic signs. Our detectors may be accellerated further by using the fast approximation of the GMM soft-assignment, as proposed in [36]. We leave this, however, for future work.

\section{Conclusion}

We have presented a novel weakly supervised localization method based on (i) Fisher embedding of local features (CNN, SIFT) and (ii) component-level sparsity induced by $\ell_{2,1}$ regularization. The Fisher embedding allows weakly 
supervised training of localization models by employing image-wide labels. The induced model sparsity reduces overfitting and enables fast evaluation by effective reduction of the representation dimensionality. This dramatically improves localization accuracy over the dense Fisher vector baseline (16 pp for zebra crossings, $21 \mathrm{pp}$ for traffic signs) and leads to a huge gain in the execution speed since our models use only a fraction of image representation (5\% for zebra crossings, $1 \%$ for traffic signs).

In order to make this approach compatible with power and metric normalizations employed in the improved Fisher vector, we have proposed two methodological novelties. First, we have introduced a first-order approximation of the normalized FV score, which allows to determine the patch-level contribution by a simple dot product. This approximation achieved comparable localization accuracy with respect to the direct approach, while gaining a huge overall execution speedup (around $200 \%$ on the traffic sign dataset). Second, we have proposed to use intra-component metric normalization in conjunction with the component-level sparsity. This setting further increased the model sparsity and reduced the localization miss frequency.

We have evaluated our method on two challenging road mapping datasets. First, we have introduced a novel dataset containing 2381 images of zebra crossings obtained by semi-automated matching of OpenStreetMap data (longitude, latitude) to GPS references of video frames. Second, we also show experimental results on an adapted version of a public traffic sign dataset [51]. Our study has shown that reliable general purpose object localization models can be trained from geo-referenced video and crowd-sourced image-wide labels provided by services such as OpenStreetMap, despite weak supervision signal provided by these resources. These localization models can be applied in automated road safety inspection and other kinds of road-environment mapping.

\section{Acknowledgments}

This work has been supported by the Croatian Science Foundation under the project I-2433-2014, the European Regional Development Fund under the grant KK.01.1.1.01.0009 (DATACROSS), and by the French national research agency (grant numbers ANR16-CE23-0006, ANR-11-LABX0025-01).

The authors would like to thank Marko Ševrović and Mario Miler for their kind assistance in accessing the employed geo-referenced videos. The authors also thank the associate editor and the anonymous reviewers for their comments and suggestions that helped improve the paper.

\section{Appendix A. Gradient of the normalized image score}

In this appendix, we derive the expression for gradient approximation given by the equation (9). We consider $\mathbf{X}$ to be an un-normalized full image Fisher vector, and $X_{d}$ to be a $d$-th dimension of the corresponding vector. The Fisher vector $\mathbf{X}$ is first normalized over each dimension $d$ with the power normalization $\mathbf{s}(\mathbf{X})=\operatorname{sign}(\mathbf{X})|\mathbf{X}|^{\rho}$. The partial derivative of the vector $\mathbf{s}(\mathbf{X})$ with respect to $X_{d}$ is given by:

$$
\frac{\partial \mathbf{s}(\mathbf{X})}{\partial X_{d}}=\left[0 \ldots \rho\left|X_{d}\right|^{\rho-1} \ldots 0\right]
$$

After the power normalization, we apply the metric normalization by dividing with the square root of $n(\mathbf{X})=$ $\mathbf{s}(\mathbf{X})^{\top} \mathbf{s}(\mathbf{X})$. Throughout this appendix, we assume the global $\ell_{2}$ normalization for the sake of simplicity. The proposed reasoning also holds for intra-component normalization, where we substitute the $n(\mathbf{X})$ with $n\left(\mathbf{X}^{k}\right)$, where $\mathbf{X}^{k}$ corresponds to the $k$-th Gaussian statistics from the descriptor $\mathbf{X}$. The partial derivative of the scalar $n(\mathbf{X})$ with respect to $X_{d}$ is given by:

$$
\begin{aligned}
\frac{\partial n(\mathbf{X})}{\partial X_{d}} & =2 s\left(X_{d}\right) \frac{\partial s\left(X_{d}\right)}{\partial X_{d}} \\
& =2 s\left(X_{d}\right) \rho\left|X_{d}\right|^{\rho-1}
\end{aligned}
$$


The classification score of the normalized image FV is computed as $f(\mathbf{X})=\mathbf{w}^{\top} \mathbf{s}(\mathbf{X}) / \sqrt{n(\mathbf{X})}$. Thus the gradient $\nabla_{X_{d}} f(\mathbf{X})$ can be expressed as:

$$
\begin{aligned}
\frac{\partial f(\mathbf{X})}{\partial X_{d}} & =\frac{\partial \mathbf{w}^{\top} \mathbf{s}(\mathbf{X}) / \sqrt{n(\mathbf{X})}}{\partial X_{d}} \\
& =\frac{1}{\sqrt{n(\mathbf{X})}} \frac{\partial \sum_{d} w_{d} \cdot s\left(X_{d}\right)}{\partial X_{d}}+\mathbf{w}^{\top} \mathbf{s}(\mathbf{X}) \frac{\partial[n(\mathbf{X})]^{-0.5}}{\partial X_{d}} \\
& =\frac{w_{d}}{\sqrt{n(\mathbf{X})}} \frac{\partial s\left(X_{d}\right)}{\partial X_{d}}-0.5 \cdot \frac{\mathbf{w}^{\top} \mathbf{s}(\mathbf{X})}{[n(\mathbf{X})]^{1.5}} \frac{\partial n(\mathbf{X})}{\partial X_{d}}
\end{aligned}
$$

When we substitute expressions for power and metric derivatives $(A .1)$ and $(\mathrm{A} .3$, we arrive to the equation 9 :

$$
\begin{aligned}
\frac{\partial f(\mathbf{X})}{\partial X_{d}} & =\frac{w_{d}}{\sqrt{n(\mathbf{X})}} \rho\left|X_{d}\right|^{\rho-1}-0.5 \cdot \frac{\mathbf{w}^{\top} \mathbf{s}(\mathbf{X})}{[n(\mathbf{X})]^{1.5}} \cdot 2 \cdot s\left(X_{d}\right) \cdot \rho\left|X_{d}\right|^{\rho-1} \\
& =\frac{\rho\left|X_{d}\right|^{\rho-1}}{\sqrt{n(\mathbf{X})}}\left(w_{d}-\frac{\mathbf{w}^{\top} \mathbf{s}(\mathbf{X})}{n(\mathbf{X})} \cdot s\left(X_{d}\right)\right) \\
& =\frac{\rho\left|X_{d}\right|^{\rho-1}}{\sqrt{n(\mathbf{X})}}\left(w_{d}-\frac{f(\mathbf{X}) s\left(X_{d}\right)}{\sqrt{n(\mathbf{X})}}\right)
\end{aligned}
$$

\section{References}

[1] M. Pandey, S. Lazebnik, Scene recognition and weakly supervised object localization with deformable part-based models, in: ICCV, 2011, pp. 1307-1314.

[2] A. J. Bency, H. Kwon, H. Lee, S. Karthikeyan, B. S. Manjunath, Weakly supervised localization using deep feature maps, in: Computer Vision - ECCV 2016 - 14th European Conference, Amsterdam, The Netherlands, October 11-14, 2016, Proceedings, Part I, 2016 , pp. 714-731.

[3] R. G. Cinbis, J. J. Verbeek, C. Schmid, Weakly supervised object localization with multi-fold multiple instance learning, IEEE Trans. Pattern Anal. Mach. Intell. 39 (1) (2017) 189-203.

[4] E. J. Crowley, A. Zisserman, Of gods and goats: Weakly supervised learning of figurative art, in: BMVC, 2013.

[5] O. Chum, A. Zisserman, An exemplar model for learning object classes, in: CVPR, 2007.

[6] C. Galleguillos, B. Babenko, A. Rabinovich, S. J. Belongie, ”Weakly Supervised Object Localization with Stable Segmentations”, in: ECCV, 2008, pp. 193-207.

[7] P. Siva, T. Xiang, "Weakly supervised object detector learning with model drift detection”, in: ICCV, 2011 , pp. $343-350$.

[8] T. Deselaers, B. Alexe, V. Ferrari, "Weakly Supervised Localization and Learning with Generic Knowledge", IJCV 100 (3) (2012) $275-293$.

[9] H. Bilen, M. Pedersoli, T. Tuytelaars, Weakly supervised object detection with convex clustering, in: IEEE Conference on Computer Vision and Pattern Recognition, CVPR 2015, Boston, MA, USA, June 7-12, 2015, 2015, pp. 1081-1089.

[10] H. Bilen, A. Vedaldi, Weakly supervised deep detection networks, in: 2016 IEEE Conference on Computer Vision and Pattern Recognition, CVPR 2016, Las Vegas, NV, USA, June 27-30, 2016, 2016, pp. 2846-2854.

[11] A. Diba, V. Sharma, A. M. Pazandeh, H. Pirsiavash, L. V. Gool, Weakly supervised cascaded convolutional networks, CoRR abs/1611.08258.

[12] M. H. Nguyen, L. Torresani, F. D. la Torre, C. Rother, "Learning discriminative localization from weakly labeled data", Pattern Recognition 47 (3) (2014) 1523-1534.

[13] M. Oquab, L. Bottou, I. Laptev, J. Sivic, Is object localization for free? - weakly-supervised learning with convolutional neural networks, in: IEEE Conference on Computer Vision and Pattern Recognition, CVPR 2015, Boston, MA, USA, June 7-12, 2015, 2015 , pp. 685-694.

[14] B. Zhou, A. Khosla, À. Lapedriza, A. Oliva, A. Torralba, Learning deep features for discriminative localization, in: 2016 IEEE Conference on Computer Vision and Pattern Recognition, CVPR 2016, Las Vegas, NV, USA, June 27-30, 2016, 2016, pp. $2921-2929$.

[15] J. Krapac, S. Šegvić, Weakly supervised object localization with large Fisher vectors, in: VISAPP, 2015.

[16] V. Zadrija, J. Krapac, J. J. Verbeek, S. Šegvić, Patch-level spatial layout for classification and weakly supervised localization, in: Pattern Recognition - 37th German Conference, GCPR 2015, Aachen, Germany, October 7-10, 2015, Proceedings, 2015, pp. 492-503.

[17] Y. Zhu, Y. Zhou, Q. Ye, Q. Qiu, J. Jiao, Soft proposal networks for weakly supervised object localization, in: IEEE International Conference on Computer Vision, ICCV, Venice, Italy, 2017, pp. 1859-1868.

[18] K. Simonyan, A. Zisserman, "Very Deep Convolutional Networks for Large-Scale Image Recognition", in: ICLR, 2015.

[19] J. Sánchez, F. Perronnin, T. Mensink, J. J. Verbeek, ”Image Classification with the Fisher Vector: Theory and Practice”, IJCV 105 (3) (2013) $222-245$.

[20] Z. Qiu, T. Yao, T. Mei,Deep quantization: Encoding convolutional activations with deep generative model CoRR abs/1611.09502. URL http://arxiv.org/abs/1611.09502

[21] M. Cimpoi, S. Maji, I. Kokkinos, A. Vedaldi, Deep filter banks for texture recognition, description, and segmentation, International Journal of Computer Vision 118 (1) (2016) 65-94.

[22] R. Jenatton, J. Mairal, G. Obozinski, F. Bach, Proximal methods for hierarchical sparse coding, J. Mach. Learn. Res. 12 (2011) $2297-2334$.

[23] D. Novotný, D. Larlus, F. Perronnin, A. Vedaldi, Understanding the fisher vector: a multimodal part model CoRR abs/1504.04763. URL http://arxiv.org/abs/1504.04763 
[24] D. Ahmetovic, R. Manduchi, J. M. Coughlan, S. Mascetti, Zebra crossing spotter: Automatic population of spatial databases for increased safety of blind travelers in: Y. Yesilada, J. P. Bigham (Eds.), Proceedings of the 17th International ACM SIGACCESS Conference on Computers \& Accessibility, ASSETS 2015, Lisbon, Portugal, October 26-28, 2015, ACM, 2015, pp. 251-258. doi:10.1145/2700648. 2809847

URL http://doi.acm.org/10.1145/2700648.2809847

[25] D. Koester, B. Lunt, R. Stiefelhagen, Zebra crossing detection from aerial imagery across countries in: K. Miesenberger, C. Bühler, P. Penáz (Eds.), Computers Helping People with Special Needs - 15th International Conference, ICCHP 2016, Linz, Austria, July 13-15, 2016, Proceedings, Part II, Vol. 9759 of Lecture Notes in Computer Science, Springer, 2016, pp. 27-34. doi:10.1007/978-3-319-41267-2_5 URL http://dx.doi.org/10.1007/978-3-319-41267-2_5

[26] Google Inc, ICMLA 2011 StreetView Recognition Challenge Online; accessed 2016-11-22 (2016).

URL http://www.icmla-conference.org/icmla11/challenge.htm

[27] Promet i Prostor, E-roads web platform Online; accessed: 2016-09-17. URL https://prometiprostor.hr/en/solutions/

[28] J. Sánchez, F. Perronnin, T. Mensink, J. Verbeek, Image Classification with the Fisher Vector: Theory and Practice, International Journal of Computer Vision 105 (3) (2013) 222-245

[29] M. Yuan, Y. Lin, Model selection and estimation in regression with grouped variables, J. R. Stat. Soc. Ser. B Stat. Methodol. 68 (1) (2006) 49-67.

[30] F. Perronnin, J. Sánchez, T. Mensink, Improving the Fisher kernel for large-scale image classification, in: ECCV, 2010 , pp. 143-156.

[31] T. Jaakkola, D. Haussler, Exploiting generative models in discriminative classifiers, in: NIPS, 1998, pp. 487-493.

[32] F. Perronnin, C. R. Dance, Fisher kernels on visual vocabularies for image categorization, in: 2007 IEEE Computer Society Conference on Computer Vision and Pattern Recognition (CVPR 2007), 18-23 June 2007, Minneapolis, Minnesota, USA, 2007.

[33] R. Arandjelović, A. Zisserman, All about VLAD, in: IEEE Conference on Computer Vision and Pattern Recognition, 2013.

[34] C. Bishop, Pattern recognition and machine learning, Springer, New York, 2006.

[35] Z. Zhang, Y. Xu, J. Yang, X. Li, D. Zhang, A survey of sparse representation: Algorithms and applications, IEEE Access 3 (2015) 490-530.

[36] J. Krapac, S. Šegvić, Fast Approximate GMM Soft-Assign for Fine-Grained Image Classification with Large Fisher Vectors, in: GCPR, 2015.

[37] P. A. Viola, M. J. Jones, Robust real-time face detection, International Journal of Computer Vision 57 (2) (2004) $137-154$.

[38] OpenStreetMap Wiki, Map Features — OpenStreetMap Wiki, online; accessed 2016-11-14 (2016). URL http://wiki.openstreetmap.org/w/index.php?title=Map_Features\&oldid=1356182

[39] Geofabrik, OpenStreetMap Data Extracts Online; accessed: 2016-11-14.

URL http://http://download.geofabrik.de/

[40] OpenStreetMap Foundation, OpenStreetMap Online; accessed 2016-11-14 (2016).

URL https: //www .openstreetmap.org

[41] OpenStreetMap Wiki, Overpass API - OpenStreetMap Wiki, Online; accessed 2016-09-17. URL http://wiki.openstreetmap.org/wiki/Overpass_API

[42] OpenStreetMap Wiki, Video mapping — OpenStreetMap Wiki, Online; accessed 2016-11-14 (2016). URL http://wiki.openstreetmap.org/w/index.php?title=Video_mapping\&oldid=1316747

[43] Mapillary, Mapillary mobile application online; accessed: 2016-11-16. URL https://play.google.com/store/apps/details?id=app.mapillary\&hl=en

[44] Telenav GmbH, OpenStreetView mobile application Online; accessed: 2016-11-16. URL https://play.google.com/store/apps/details?id=com.telenav.streetview\&hl=en

[45] M. Everingham, L. Gool, C. K. Williams, J. Winn, A. Zisserman, The Pascal visual object classes (VOC) challenge, Int. J. Comput. Vision 88 (2) (2010) 303-338.

[46] K. Chatfield, V. S. Lempitsky, A. Vedaldi, A. Zisserman, The devil is in the details: an evaluation of recent feature encoding methods, in: British Machine Vision Conference, BMVC 2011, Dundee, UK, August 29 - September 2, 2011. Proceedings, 2011 , pp. 1-12.

[47] R. M. French, Catastrophic forgetting in connectionist networks: Causes, consequences and solutions, Trends in Cognitive Sciences 3 (4) (1999) 128-135.

[48] M. Douze, H. Jégou, The Yael library, in: Proceedings of the ACM International Conference on Multimedia, 2014.

[49] J. Mairal, F. Bach, J. Ponce, G. Sapiro, Online learning for matrix factorization and sparse coding, J. Mach. Learn. Res. 11 (2010) 19-60.

[50] Inland transport comitee, Convention on road signs and signals (1968).

[51] K. Brkić, A. Pinz, S. Šegvić, Z. Kalafatić, Histogram-based description of local space-time appearance, in: SCIA, 2011, pp. 206-217.

[52] N. Dalal, B. Triggs, Histograms of oriented gradients for human detection, in: CVPR, 2005. 